COMbined Microbial SURFACTANT-POL YMER SySTEM FOR IMPROVED OIL MOBILITY AND CONFORMANCE CONTROL

FINAL PROGRESS REPORT

PERIOD: SET. 2001-AUG. 2005

CONTRACT NUMBER: DE-FG26-01NT41357

PROJECT START DATE:

PROJECT DURATION:

TOTAL FUNDING REQUESTED:

FIRST YEAR REQUEST:
September 2001

September 2001 - August 2005

$\$ 199,320$

$\$ 88,900$

TECHNICAL POINTS OF CONTACT:

Jorge Gabitto

Prairie View A\&M State University

Department of Chemical Engineering

Prairie View, TX 77429

TELE:(936) 857-2427

FAX: (936) 857-4540

EMAIL:jgabitto@aol.com
Maria Barrufet

Texas A\&M University

Petroleum Engineering

Department

College Station TX, 77204

TELE:(979) 845-0314

FAX:(979) 845-0325

EMAIL:barrufet@spindletop. tamu.edu 


\section{COMBINED MiCROBIAL SURFACTANT-POLYMER SYSTEM FOR IMPROVED OIL MOBILITY AND CONFORMANCE CONTROL}

\section{DISCLAIMER}

This report was prepared as an account of work sponsored by an agency of the United States Government. Neither the United States Government nor any agency thereof, nor any of their employees, makes any warranty, express or implied, or assumes any legal liability or responsibility for the accuracy, completeness, or usefulness of any information, apparatus, product, or process disclosed, or represents that its use would not infringe privately owned rights. Reference herein to any specific commercial product, process, or service by trade name, trademark, manufacturer, or otherwise does not necessarily constitute or imply its endorsement, recommendation, or favoring by the United States Government or any agency thereof. The views and opinions of authors expressed herein do not necessarily state of reflect those of the United States Government or any agency thereof. 


\title{
COMBINED MiCROBIAL SURFACTANT-POLYMER SYSTEM FOR IMPROVED OIL MOBILITY AND CONFORMANCE CONTROL
}

\begin{abstract}
Many domestic oil fields are facing abandonment even though they still contain twothirds of their original oil. A significant number of these fields can yield additional oil using advanced oil recovery (AOR) technologies. To maintain domestic oil production at current levels, AOR technologies are needed that are affordable and can be implemented by the independent oil producers of the future.

Microbial enhanced oil recovery (MEOR) technologies have become established as cost-effective solutions for declining oil production. MEOR technologies are affordable for independent producers operating stripper wells and can be used to extend the life of marginal fields. The demonstrated versatility of microorganisms can be used to design advanced microbial systems to treat multiple production problems in complex, heterogeneous reservoirs.

The proposed research presents the concept of a combined microbial surfactantpolymer system for advanced oil recovery. The surfactant-polymer system utilizes bacteria that are capable of both biosurfactant production and metabolically-controlled biopolymer production. This novel technology combines complementary mechanisms to extend the life of marginal fields and is applicable to a large number of domestic reservoirs. The research project described in this report was performed by BioEngineering Inc., a woman owned small business, Texas A\&M University and Prairie View A\&M University, a Historically Black College and University.

This report describes the results of our laboratory work to grow microbial cultures, the work done on recovery experiments on core rocks, and computer simulations. We have selected two bacterial strains capable of producing both surfactant and polymers. We have conducted laboratory experiments to determine under what conditions surfactants and polymers can be produced from one single strain. We have conduct recovery experiments to determine the performance of these strains under different conditions. Our results show a significant influence of nutrient regime on alternate production of surfactants or polymers for a Bacillus licheniformis strain, NIPER 1A.

The main conclusion of this work is that NIPER 1A can produce both surfactant and polymer by nutrient regime manipulation. Our experiments proved that this property leads to improved oil recovery by increasing alternatively, oil mobility and conformance control.
\end{abstract}




\section{COMBINED MicROBIAL SURFACTANT-POLYMER SYSTEM FOR IMPROVED OIL MOBILITY AND CONFORMANCE CONTROL}

\section{TABLE OF CONTENTS}

DISCLAIMER 2

ABSTRACT 3

TABLE OF CONTENTS 4

STATEMENT OF WORK 6

$\begin{array}{lc}\text { TECHNICAL DESCRIPTION } & 7\end{array}$

$\begin{array}{ll}\text { INTRODUCTION } & 7\end{array}$

$\begin{array}{ll}\text { Enhanced Oil Recovery (MEOR) } & 7\end{array}$

$\begin{array}{ll}\text { Biosurfactants } & 10\end{array}$

$\begin{array}{ll}\text { Biopolymers } & 12\end{array}$

Goals 13

$\begin{array}{ll}\text { EXPERIMENTAL SECTION } & 14\end{array}$

$\begin{array}{lr}\text { Growth Experiments } & 14\end{array}$

$\begin{array}{lr}\text { Introduction } & 14\end{array}$

$\begin{array}{ll}\text { Bacterial Strains } & 15\end{array}$

$\begin{array}{ll}\text { Measuring Techniques } & 17\end{array}$

$\begin{array}{ll}\text { Colony Counting Procedures } & 17\end{array}$

$\begin{array}{lr}\text { Surface Tension Measurements } & 18\end{array}$

$\begin{array}{lr}\text { Viscosity Measurements } & 19\end{array}$

Chemical Analysis 19

$\begin{array}{lr}\text { Porous Media Tests } & 20\end{array}$

$\begin{array}{lr}\text { SIMULATION SECTION } & 22\end{array}$

$\begin{array}{lr}\text { Simulator Background } & 22\end{array}$

Mass Conservation Equations 23

Pressure Conservation Equations $\quad 24$

Fluid and Soil Properties $\quad 24$

Polymer Adsorption $\quad 25$ 
$\begin{array}{ll}\text { Viscosity } & 25\end{array}$

Microbial Enhanced Oil Recovery Model 26

$\begin{array}{ll}\text { Surfactant/Brine/Oil Phase Behavior } & 27\end{array}$

TASK DESCRIPTION 28

Task 1 - Characterization of Surfactant and Polymer-Producing $\begin{array}{ll}\text { Bacteria } & 28\end{array}$

Subtask 1.1 - General Bacterial Physiology 28

Subtask 1.2 - Surfactant Production 30

Subtask 1.3 - Polymer Production 32

Task 2 -Evaluation of Nutrient Regimes 33

Subtask 2.1 - Polymer Stimulation 33

Task 3 -Testing of Microbial Systems in Porous Media 36

Subtask 3.1 - Microbial Transport 36

Subtask 3.2 - Improved Oil Mobility 38

Subtask 3.3 - Improved Sweep Efficiency 4

Task 4 -Process Simulation $\quad 43$

Subtask 4.1 - UTCHEM Simulator 43

$\begin{array}{ll}\text { CONCLUSIONS } & 46\end{array}$

REFERENCES

TABLES AND FIGURES

$\begin{array}{ll}\text { PROJECT INFORMATION } & 67\end{array}$

$\begin{array}{lr}\text { PUBLICATIONS AND PRESENTATIONS } & 67\end{array}$

$\begin{array}{lr}\text { STUDENTS } & 67\end{array}$ 


\section{STATEMENT OF WORK (SOW)}

Under this Statement of Work (SOW), Dr. Jorge Gabitto from the Chemical Engineering Department at Prairie View A\&M University (PVAMU), Dr. Maria Barrufet from the Petroleum Engineering Department at Texas A\&M University (TAMU) and Dr. Rebecca Bryant from Bio-Engineering International Inc. (BEI) conducted research and training in the area of microbial improved oil recovery techniques.

A research project was proposed to develop and prove the concept of a combined surfactant-polymer microbial system for advanced oil recovery. The surfactant-polymer system utilizes bacteria that are capable of both biosurfactant production and metabolically-controlled biopolymer production. This novel technology combines complementary mechanisms to extend the life of marginal fields and is applicable to a large number of domestic reservoirs.

This research involved training of graduate and undergraduate students in state of the art techniques. Technology transfer of the results generated by the project is achieved through Dr. Bryant's efforts, presentations on scientific/professional meetings, and publications in refereed journals.

Dr. Gabitto acted as coordinator of the research team, conducted numerical simulations using commercial software, and he was responsible for part of the experimental program. Dr. Barrufet, Co-Principal Investigator, was responsible for part of the experimental part. Dr. Bryant provided the microorganisms to be used in thee project, advised the research team, and was responsible for transferring the project's findings to small independent producers. 


\section{TECHNICAL DESCRIPTION}

\section{INTRODUCTION}

This report has been organized in a way that tries to maximize the amount of information while minimizing the length. It starts with a brief introduction and a statement of goals. An experimental part that tries to describe in a general way the techniques used throughout the project follows. Technical introduction and explanations of procedures are included in this section. A description about the commercial program (UTCHEM) used for simulation calculations is also attached. All the project results are listed in a section that describes the different specific tasks that comprise the project. Specific details related to experimental work are also included in the tasks description. The technical part concludes summarizing the project's main findings and conclusions.

A list of publications/presentations related to the project is attached in a separate section. A brief description of the students participating in the project and their respective tasks is also included.

\section{Enhanced Oil Recovery (MEOR)}

The most common EOR methods are the chemical and the physical methods ${ }^{1}$. Chemical methods of enhanced oil recovery are characterized by the addition of chemicals to water in order to generate fluid properties or interfacial conditions that are more favorable for oil displacement. Polymer flooding, using polyacrylamides or polysaccharides, is conceptually simple and inexpensive, and its commercial use is increasing despite the fact that it raises potential production by only small increments. Surfactant flooding is complex, requiring detailed laboratory testing to support field projects. It is also expensive and is only used in few large-scale projects. .

Microbial Enhanced Oil Recovery (MEOR) is an EOR method that uses microorganisms and their metabolic products to improve oil production in the reservoir. The majority of the MEOR work leading to field trials has been completed in about the last 15 years. The technology has advanced from a laboratory-based evaluation of microbial processes, to field applications internationally. MEOR has been recognized as 
a potentially cost-effective method, particularly for stripper wells (well that produces less than $10 \mathrm{bbl} /$ day) ${ }^{2}$.

The concept of using microorganisms and their activities in an enhanced oil recovery procedure is not new. The first suggestion for using MEOR was made as early as in 1926, when Beckman reported the action of bacteria on mineral oil ${ }^{3}$. As a result of his work, he proposed that bacterial enzymes could be used in oil recovery. However, little work was done until ZoBell started a series of systematic laboratory investigations in the 1940 's in connection with the American Petroleum Institute ${ }^{4-6}$. The results and ideas presented in ZoBell's articles marked the beginning of a new era of research in petroleum microbiology. Much more experimental work will have to be done by trained microbiologists in cooperation with petroleum engineers before any definite recommendations can be made.

A number of field trials were initiated during 1980's and 1990's. The conventional method used was to inject an adapted mixture of bacteria into the reservoir together with a cheap carbon source such as molasses. This process is limited to small onshore installation due to the amount of molasses needed. In later years considerable work has been done in analyzing the potential for doing MEOR on larger offshore fields ${ }^{7}$.

In this study we concentrated on microbial enhanced waterflooding. MEOR is applied to existing waterfloods to improve their performance and enhance oil production by treating the entire reservoir. MEOR materials are added, either continuously or periodically, to the water holding tanks at the primary injection stations. Normal waterflooding operations are not interrupted when the MEOR process is initiated in a field.

In conventional MEOR methods a special culture of microorganisms is injected into the reservoir. This bacterial culture is selected for each individual reservoir and it could be injected as a pure culture, a mixed culture or an adapted culture. During the process, a complete growth medium containing all major nutrients necessary for microbial growth including carbon, nitrogen, and phosphorus, is injected into the reservoir to feed the injected microorganisms. The microorganisms replicate and produce chemicals for oil mobilization based entirely on the cost of nutrients. Bryant et al. ${ }^{8}$ presented the results of two microbial enhanced waterflood fields performed by NIPER (National Institute for 
Petroleum and Energy Research) at the Mink Unit site, Delaware, Oklahoma. After this pilot project demonstrated a $13 \%$ increase in oil production, an expanded field pilot program at the Phoenix field site in the same area was initiated. The Phoenix field showed a $19.6 \%$ improvement in oil production, and using the nutrient cost only, $\$ 2.33 /$ incremental bbl of oil was calculated. The cost for the Mink unit site was calculated to be $\$ 3.23 /$ incremental bbl. of oil. Unlike commercial surfactant processes, which are linked to the cost of the chemicals and energy involved in their manufacture, microbial surfactant can be produced using inexpensive feedstock ${ }^{8}$. The microorganisms can be regulated by the amount of nutrient present. In other words, if they are not fed, they will disappear.

In the use of microorganisms in-situ for MEOR, it is necessary to use microbial cultures that can survive in the reservoir environment, but also produce the chemicals in sufficient quantities that are necessary for oil mobilization. Before the bacteria are injected into the reservoir they must have very good growth under facultative anaerobic conditions (e.g., can survive under both aerobic and anaerobic conditions), and a high metabolic activity of molasses with important production of oil displacement agents ${ }^{9}$.

Laboratory research has demonstrated that microbial products can change the chemical and physical properties of oil, and selectively plug high permeability zones to improve sweep efficiency ${ }^{10-12}$. The transport of microorganisms in the reservoir rock has also been studied in the laboratory and the results indicate that certain strains of microbes can be transported through the reservoir rock under proper conditions. Associated with these laboratory investigations mathematical simulations have been introduced to help understand the mechanisms involved in the MEOR process ${ }^{9,13}$.

The microorganisms play various roles in the reservoir. The most important ones considered in this project are: production of surfactants, selective plugging of the reservoir, polymer production, and alcohol production ${ }^{14}$. Microorganisms can produce surfactants that can decrease surface and oil water interfacial tension to as low as $5 * 10^{-3}$ $\mathrm{mN} / \mathrm{m}^{15}$. Interfacial tension between oil and water is normally about $10 \mathrm{mN} / \mathrm{m}^{15}$. In general, a biosurfactant is easily dissolved in connate water or injection water and acts favorable on the interface between oil and water. Interfacial tension reduction decreases the pressure required to release oil trapped in the rock pores by capillary forces, which 
displaces oil from the pores into the mobile liquid phase. Microbial biosurfactants usually act in the same way as synthetic surfactants, and have the same characteristics ${ }^{15}$.

Another application for microorganisms in a waterflood is fluid diversion. Since many types of microorganisms produce polymers, biomass and slimes, it has been suggested that some microorganisms could be used in-situ to preferentially plug high permeability zones in the reservoir, and thus improve sweep efficiency ${ }^{16-17}$.

Microorganisms can also produce organic solvents like alcohol. Solvents decrease oil viscosity, making it thinner and increasing flow ${ }^{14}$.

\section{Biosurfactants}

Biosurfactants are surface active agents produced by certain types of microorganisms during their growth on insoluble substrates. Microbial compounds, which exhibit particularly high surface activity are classified as biosurfactants. (Cooper ${ }^{18}$ ) Many species have the capabilities to degrade hydrocarbons and to synthesize biosurfactants (Syldatk and Wagner ${ }^{19}$; Riser-Roberts ${ }^{20}$ ).

There are five major kinds of biosurfactants that are widely used, they are, glycolopids; phospholipids and fatty acids; lipopeptide-lipoproteins; polymeric surfactants; and particulate surfactants ${ }^{21}$. The more relevant surfactants to this study are the lipopeptide surfactants.

Glycolipids are the most commonly isolated and studied biosurfactants, they are carbohydrates in combination with long-chain aliphatic acids or hydroxyl aliphatic acids. The best examples of glycolipids studied from the point of view of surfactant characterization and properties are rhamnolipids. Certain species of Pseudomonas (Pseudomonas aeruginosa) are known to produce large amounts of glycolipids containing one or two molecules of rhamnose linked to one or two molecules of $\beta$ hydroxydecanoic acid units ${ }^{22}$.

Lipopetide antibiotics (Polymyxins), and Decapeptide antibiotics (Gramicidins) show remarkable surface-active properties. Some peptide-containing lipids also exhibit biosurfactant activity. They include ornithine-containing lipids from P. rubescens; and $t$. thiooxydans; cerlipin; ornithine-taurine-containing lipid for Gluconobacter cerinus 
IFO3267; lysine-containing lipids from Agrobacterium tumefaciens; and streptomyces sioyaensin ${ }^{21}$.

Surfactin, a cyclic lipopetide, reported first by Arima et al. ${ }^{23}$ in B. subtilis ATCC21332, is one of the most effective biosurfactants known so far. It is capable of lowering the surface tension from 72 to $27 \mathrm{mN} / \mathrm{m}$, at a concentration as low as $0.005 \%$. The ability of surfactin to lyse red blood cells is of limited use, but this discovery has led to the development of a quick method for the screening of biosurfactant producing microbes ${ }^{23}$.

Microbial biosurfactants include a wide variety of chemical structures, such as glycolipids (Zajic et al. ${ }^{24}$, Finnerty et al. $^{25}$ ), lipopeptides (Horowitz et al. ${ }^{26}$ ), polysaccharide-protein complexes (Zajic et al. ${ }^{24}$ ), phospholipids (Beebe, and Umbreit ${ }^{27}$ ), fatty acids and neutral lipids $\left(\mathrm{Cooper}^{18}\right)$. Most biosurfactants consist of distinct hydrophilic and hydrophobic moieties. The former can be either ionic or nonionic and consist of mono-, di-, or polysaccharides, carboxylic acids, amino acids, or peptides. The hydrophobic moieties are usually saturated, unsaturated or hydroxylated fatty acids. For some high molecular weight surfactant molecules, such as protein-polysaccharide complexes, different molecules contribute the hydrophilic and hydrophobic moieties. The most widespread microbial surfactants are glycolipids. The rhamnolipids of Pseudomonoas aeruginosa and the trehalose lipids of Rhodococcus erythropolis are among the best studied biosurfactants. For both compounds the chain length of the hydrophobic domain and the structure of the carbohydrate head group exhibit considerable variability depending on the growth conditions. Surfactin consists of 3hydroxyl- 13 -methyl- tetradecanoic acid amidated to the N-terminal amine of heptapeptide. The carboxyl terminal end of the peptide is further esterified to the hydroxyl of the fatty acid. The ionizable side chains of glutamic. and aspartic acids contribute significantly to the excellent surface active properties of the molecule.

The proper characterization of the activity of biosurfactants requires detailed information on the thermodynamic properties of aqueous/non-aqueous surfactant mixtures. This information is experimentally difficult to obtain and is not available for any natural products. As a result the most frequently used indices for the performance of biosurfactants are the surface and interfacial tension and the critical micelle concentrations. 
Several biosurfactants show low CMC values, reduce the surface tension of the fermentation broth to less than $30 \mathrm{mN} / \mathrm{m}$, and the interfacial tension against n-alkanes to values below $1 \mathrm{mN} / \mathrm{m}$ (Guerra-Santos ${ }^{22}$ ). In particular the glycolipids produced by Rhodococcus sp. H13 (Finnerty and Singer ${ }^{25}$ ), and the major surfactant from Bacillus Licheniformis strain JF-2 have been shown to reduce the surface tension of aqueous solutions to $26-27 \mathrm{mN} / \mathrm{m}$ and the interfacial tension against octane to $10^{-2} \mathrm{mN} / \mathrm{m}^{28}$. These values compare favorably to those obtained with commercial synthetic surfactants. It is important to note that in addition to the major biosurfactants both Rhodococcus $s p$. H13A and Bacillus Licheniformis JF-2 (ATCC 39307) produce several additional surface active agents which appear to act synergistically (Javaheri et al. $^{28}$ ). Furthermore, some biosurfactants also exhibit good thermal and chemical stability at temperature up to $75{ }^{\circ} \mathrm{C}$ for at least 140 hours. The surfactants are stable at $\mathrm{pH}$ values between 5.5 and 12 . The surfactants slowly lose activity under more acidic conditions.

The interfacial properties of surfactants depend on the ionic composition of the aqueous phase. Salinity and divalent cations $\left(\mathrm{Mg}^{2+}, \mathrm{Ca}^{2+}\right.$, etc.) have significant influence on surfactant production and phase behavior. High concentration of $\mathrm{NaCl}$ inactivates the glycolipids of apicola. On the other hand, the interfacial tension of the fermentation broth of Bacillus Licheniformis JF-2 decreases by more than an order of magnitude in the presence of $10 \mathrm{wt} \% \mathrm{NaCl}$, but is not affected by calcium salts (McInerney et al. ${ }^{29}$ ). Interestingly, this microorganism grows and produces surfactant under both aerobic and anaerobic conditions and in the presence of up to 10 percent $\mathrm{NaCl}^{28,29}$.

\section{Biopolymers}

Microbial polymer production offers a relatively inexpensive, environmental friendly method for conformance control. Microorganisms can be used to selectively plug high permeability zones and improve sweep. Effective permeability is reduced by biomass

growth and in-situ polymer production ${ }^{12}$. Step et al. ${ }^{12}$ used a polymer producing microorganism designated as NIPER 11, which is most probably a strain of Leuconostoc mesenteroides, to study conformance control. This microorganism ferments sucrose and produces lactic acid, acetic acid, ethanol, and a dextran polymer. The biopolymer is insoluble in the culture medium and does not produce significant viscosity increase. The 
same microorganism produces copious amount of the same biopolymer using molasses as carbon source ${ }^{12}$. Jenneman et al..$^{30}$ used NIPER 11 and another strain of Leuconostoc mesenteroides to produce rigid, bulk dextran gels using molasses as carbon source. Bulk dextran gels resulted in in-depth permeability reductions higher than $90 \%$.

Some strains of Bacillus licheniformis are known to produce biopolymers. Step et al. ${ }^{12}$ reported a polymer-producing strain of Bacillus licheniformis, designated as $\mathrm{TG}_{2}-32$, isolated from arid conditions in Arizona. This microorganism produces an external, insoluble levan polymer. The polymer is a fructran polymer where the fructose units are mainly linked by $\alpha-1-6$ bonds. The average molecular weight is around 1000 .

Ramsay et al. ${ }^{31}$; used a Bacillus licheniformis, strain (NRC 9012) to produce an insoluble levan polymer which has potential applications as a selective plugging agent in microbial enhanced oil recovery. The microorganism grows in sucrose, glucose, and fructose, but produces polymer only on sucrose. Polymer production can be metabolically activated by the use of nitrate salts as nitrogen source.

\section{Goals}

The main goal of the research described in this report is to develop a combined microbial surfactant-polymer system using bacteria that produce both biosurfactant and biopolymer. The analysis has been reduced to two different bacterial strains that were judged capable of producing biosurfactants and biopolymers. One is Bacillus licheniformis (NIPER 1A), which is a well-known surfactant producer (Bryant et al. ${ }^{32}$, Lin et $\mathrm{al}^{33}$ ). The other is a laboratory modified polymer producing, Leuconostic mesenteroides (NIPER 11) ${ }^{12,30}$.

Chemical analysis, physical properties determination and recovery experiments have been used to determine the feasibility of using at least one as a surfactant/polymer producer. 


\section{EXPERIMENTAL SECTION}

\section{Growth Experiments}

\section{Introduction}

The main goal of the research described in this proposal is to develop a combined microbial surfactant-polymer system using bacteria that produce both biosurfactant and biopolymer. This concept is based on our experience with a bacterium, Bacillus licheniformis, which is known to produce both products in sufficient quantities to make the process feasible. Strains of Bacillus licheniformis have been previously reported both for surfactant production (Bryant et al. ${ }^{32}$, Lin et al. ${ }^{33}$ ) and for polymer production; (Ramsay et al. $^{31}$; Bae et al. ${ }^{34}$ ) there are no reports in the literature of the two products being investigated together for a combined process. A developed strain of $B$. licheniformis, NIPER 1A, is used. NIPER 1A transports well through oil reservoirs and produces copious amounts of biosurfactants. However, NIPER 1A produces only minimal amounts of biopolymer using common nutrients. Ramsay et al. ${ }^{31}$ have reported that the presence of ammonium ion seems to be an inhibitor to polymer production leading the organism into a metabolic path that leads to surfactant-only production. Detailed analysis of the relevant literature ${ }^{31,32,33,35}$ points also to the metabolic role played by several enzymes and additives such as trypticase, tryptone, soytone, yeast extract, etc.

Biosurfactants cause a decrease in the interfacial tension between oil and water phases, which is the primary mechanism by which surfactant-producing bacteria mobilize oil. NIPER 1A strains that produce large amounts of biosurfactant are being tested for their effects on interfacial tension between aqueous and oil phases. Interfacial tension values are measured at various stages of culture growth and over a reasonable range of nutrient levels using appropriate laboratory equipment such as spinning drop or the $d u$ Nuoy ring methods.

Polymer-producing bacteria polymerize carbohydrates present in growth substrate into extra-cellular polysaccharide biopolymers. The production of soluble biopolymers causes viscosity increases in the microbial growth medium. Polymer production can be stimulated by nutrient manipulations and/or other external factors. Optimum polymer 
production is determined by chemical assays and by measuring viscosity increases with a tubular viscometer.

An important part of this research is to validate the concept that a microbial surfactant-polymer system can be designed to advance current technology for improving oil recovery from porous media in the laboratory. We do this in a series of experiments designed to show advantages of concerted microbial polymer system blockage of watered out high permeability zones and microbial surfactant-improved mobilization of oil from oil-bearing zones. The two mechanisms for oil recovery that are developed in this research program, increased oil mobility and profile modification, are tested individually in coreflood experiments. The processes can then be combined in more complex models to test the performance of the combined system under simulated reservoir conditions.

The majority of pure culture studies have used glucose as the substrate. When grown in a batch culture, the change in microbial population with time follows the classical growth curve (Fuyimoto, $1963^{36}$ ). This curve has three quite distinct phases, namely lag, exponential and stationary. The lag phase of growth represents the acclimation period of an organism to its new environment. After the onset of cell division the organism moves out of the lag period and continues dividing exponentially until such time as the medium is no longer able to support growth. This exponential phase of growth is the most important part of the growth curve as it represents the maximum rate of substrate removal, and in wastewater treatment the aim is to remove the substrate as rapidly as possible (Gaudy and Gaudy ${ }^{37}$ ). After a given period of time the microorganism population reaches the stationary phase of growth and it is reasonable to assume that this is a direct result of substrate depletion. In this case glucose and/or other sugars are said to be the growth limiting substrate. The decline in population appearing in the last portion of the growth curve represents the death period. In this period population decline outnumbers population growth and the cells still alive remain dormant.

\section{Bacterial Strains}

The work done in the first two years of this project showed that NIPER 1A, a laboratory modified strain of Bacillus licheniformis that produces copious amount of surfactant, and NIPER11, a laboratory modified polymer-producing strain of Leuconostic 
mesenteroides; are the microorganisms relevant to this study. Therefore, the experimental work is related only to them.

Microorganisms require suitable nutrients as well as a favorable environment for growth. First, the culture medium must contain those nutrients essential for the growth of a given microorganism. Second, this medium must provide suitable surroundings for growth, the proper $\mathrm{pH}$, osmotic pressure, oxygen, temperature, etc. Many different substances will serve satisfactorily as a culture medium. We have used in this work only liquid cultures. Bryant et al. ${ }^{32}$ used also agar media prepared in petri dishes.

The microorganisms listed above were grown under different conditions in different media. Two broths, Thioglycolate by Difco, and Tryticase soy broth by Difco, were selected as the most suitable media to grow and maintain the aforementioned organisms. These broths contain meat extract and dextrose as principal nutrients plus phosphorus and nitrogen compounds. Good growth was achieved working under anaerobic or oxygen limiting conditions. The growth under aerobic conditions was significantly less and in some strains almost negligible. The original microorganisms were provided in these cultures; therefore, it was convenient to keep the original cultures in these broths, placed them in an oven at constant temperatures of $33{ }^{\circ} \mathrm{C}$. In order to study effect of nutrients on the microorganisms a medium E (ATCC 1502) complete or modified was also used. The composition of all these media is summarized in Table 1. The cultures used in growth experiments were prepared by taking a $10 \mathrm{ml}$ sample, and placing it in 250 conical flasks filled with a $1 \%$ peptone in deionized water solution. Later, sugars were added to study the influence of different sugar sources. Sucrose, glucose, and commercial molasses were added. Typically, $10 \%$ sugar solutions in peptone water were prepared. These solutions will be referred as, sucrose -peptone broth, glucose -peptone broth, etc.

Growth conditions for NIPER 1A have been published in literature ${ }^{35}$. NIPER 1A was observed to be limited to a salinity range of 0 to $5 \mathrm{wt} \% \mathrm{NaCl}$, with an upper temperature limit of $35^{\circ} \mathrm{C}$. This microbial system can tolerate a $\mathrm{pH}$ range from 4.5 to 8.0. Other microbial strains were observed to tolerate a salinity up to $14 \mathrm{wt} \% \mathrm{NaCl}$, when grow in Tryptic soy broth, and up to $5 \mathrm{wt} \%$ when grown in sucrose peptone broth. Its upper temperature limit was $45^{\circ} \mathrm{C}$, with a $\mathrm{pH}$ tolerance from 6.0 to 10.0. Optimum growth was achieved around $\mathrm{pH} 7$. 


\section{Measuring Techniques}

A brief description of the laboratory techniques used in this project is given below.

\section{Colony Counting Procedures}

In this work, the Standard Plate count technique $\left(\mathrm{SPC}^{38}\right)$ was used to enumerate the microbial population. It is a direct quantitative measurement of the viable aerobic and facultative anaerobic bacteria in a water environment, capable of growth on the selected plating medium. An aliquot of the water sample is seeded into a sterile petri dish where a tempered agar medium has been added. The plate is rotated to evenly distribute the bacteria. Each colony that develops on or in the agar medium originates theoretically from one single bacteria cell.

Although no one set of plate count conditions can enumerate all organisms present but the number and types of bacteria that developed are influenced by the time and temperature of incubation, the level of oxygen, the presence of specific nutrients in the growth medium, the $\mathrm{pH}$ of the medium and competition among cells for nutrients. The Standard Plate Count Method provides the uniform technique required for comparative testing water quality in most situations. When the method applies to research work, however, the consistency of the parameter and media becomes more important.

The preparation procedure used was, dissolve $1 \mathrm{~g}$ of peptone water (crystals) into 1 liter of distilled water, put $9 \mathrm{ml}$ to each test tube. Autoclave at $121-124{ }^{\circ} \mathrm{C}$ for 15 minutes and allow to cool to room temperature. Dissolve 23 grams of Nutrient Agar inside 1 liter of water and boil completely. Autoclave the solution at $121-124{ }^{\circ} \mathrm{C}$ for 15 minutes, and place agar in a tempering water bath maintained at a temperature of $44-46{ }^{\circ} \mathrm{C}$. Do not hold agar at this temperature longer than three hours because it may form precipitates which confuse the counting of colonies.

Put $9 \mathrm{ml}$ of $0.1 \%$ peptone water in 10 different sterile test tubes, label them as $10^{-1}$, $10^{-2}, \ldots, 10^{-10}$, make a $1: 10$ dilution using $1 \mathrm{ml}$ of solution A with a $1 \mathrm{ml}$ pipette, mix each tube carefully by vortexing. Obtain 11 sterile petri dishes, label them with the appropriate dilution i.e. $1,10^{-1}, \ldots 10^{-10}$. Begin with highest dilution, and working backward, pipet $1.0 \mathrm{ml}$ of aliquot from every diluted sample into their appropriate petri dishes including the straight sample. Pour approximately $10 \mathrm{ml}$ of nutrient agar (held at 
$45{ }^{\circ} \mathrm{C}$ ) into each petri dish, swirl gently to disperse the sample evenly, put on all the cover of the petri dishes, allow to cool down for 10 minutes. After the agar plates have hardened on a level surface, invert the plates and immediately incubate at $35{ }^{\circ} \mathrm{C}$ for 24 48hrs. Also put $1 \mathrm{ml}$ of peptone water into a petri dish, add agar mix and incubate with the others. This is the control plate, it will check the sterility of pipets, agar, dilution peptone water, and petri dishes.

After the required incubation period, examine plates and select those with 30-300 colonies. Count these plates immediately by placing the plate to be counted on a colony counter, a standard plastic petri dish has a surface area of $57 \mathrm{~cm}^{2}$; count the colonies in 13 squares usually highlighted on the surface of the colony counter a representative distribution of colonies. Select 7 consecutive horizontal squares and 6 consecutive vertical squares for counting, some colonies will contact with grid lines, count the colonies individually, even if they are in contact with each other.

Record the sum of the colonies in these $13 \mathrm{~cm}^{2}$, and multiply by 4.32 to estimate the colonies per plate. Then multiply by dilution to determine the count/ml, i.e., the number of bacteria/ml of your original sample, record as S.P. Count $/ \mathrm{ml}$ (Standard Plate Count per $\mathrm{ml}$ ). The count is also referred to as $\mathrm{cfu} / \mathrm{ml}$ (Colony Forming Units per $\mathrm{ml}$ ).

If there are less than 30 colonies on all plates, record the actual number of colonies on the lowest dilution plated and report the count as, Estimated Standard Plate Count per milliliter e.g. $\mathrm{ESPC}=100 \mathrm{cfu} / \mathrm{ml}$. If $1 \mathrm{ml}$ volumes of original sample produce counts less than 30, actual counts are reported.

\section{Surface Tension Measurements}

Measurements of the surface tension of the different cultures were made using a Fisher model 21 tensiomat tensiometer. The apparatus is based on the du Nuoy ring method. In this method a ring (standard perimeter about $60 \mathrm{~mm}$ ) is pulled through the liquid/air interface and the maximum downward force directed to the ring is measured. Note that the force will slightly decrease before the ring is ruptured to the air. This method is not direct. One must use correction factors which take account the dimensions of the ring (the perimeter, ring wire thickness and the effect of the opposite inner sides of 
the ring to the measurement). All measurements were made at a temperature range of 20 $23^{\circ} \mathrm{C}$.

A variation of this procedure was introduced in order to measured low interfacial tensions between the microbial solution and an oil phase. The method was based on the procedure reported by Tsouris ${ }^{39}$ (2003). In this procedure a hydrocarbon (oil) phase is placed on top of the microbial solution. The force needed to lift the platinum ring through the interface between the microbial solution and the hydrocarbon phase gives a direct measurement of interfacial tension. This method allows measurement of interfacial tensions as low as $1 \mathrm{mN} / \mathrm{m}$.

A spinning drop tensiometer available at the Petroleum Engineering Department at TAMU was used in some measurements of very low interfacial tension values. This is a method used when the surface tension values are so low that normal tensiometers, which use other methods, are not able to make the measurement. A small drop of a sample is injected inside a thin tube with another liquid. The tube is then spinned at a high speed and the interfacial surface tension is calculated from the angular speed of the tube and the shape of the drop. The denser medium, the culture, is used as the continuous phase. One oil drop is introduced into the continuous phase.

\section{Viscosity Measurements}

Viscosity was measured using an Ube-Lode type viscometer. The apparatus allows viscosity measurements in the range $1-10 \mathrm{cp}$ at $25{ }^{\circ} \mathrm{C}$. Only $2 \mathrm{ml}$ of sample are needed for the measurements. The apparatus is based on the Hagen-Pousielle equation that predicts a linear relationship between a fluid viscosity and the time required for this fluid to transverse the distance between two fixed marks on a cylindrical calibrated glass tube.

\section{Chemical Analysis}

Most of the prepared bacterial samples were kept under incubation for several months. The polymer producing strains produced a copious amount of biopolymers during that time. A white solid was clearly visible by the bottom of the assay tubes commonly used during this research. NIPER 11 samples presented this behavior for all 
samples. The precipitate was even noticeable in the prepared samples after incubation for less than one week in an anaerobic environment. In cases where this behavior was not appreciated alcohol solubility test were conducted. These simple tests are based on the insolubility of biopolymers in an alcohol-water medium. The cells were removed from the culture by centrifugation at $15,000 \mathrm{~g}$ for 15 minutes. Up to $3 \mathrm{~V}$ of methyl alcohol is added to the assay tube until an alcohol-water medium is prepared. The change in medium polarity produces precipitation of several biopolymers. A cloudy-white coloration is indicative of polymer production. After incubation for several days a white precipitate appears by the bottom of the essay tube.

\section{Porous Media Tests}

One of the purposes of the microbial polymer systems for improving oil recovery is to block fluid flow through watered out thief zones in the reservoir and divert fluid flow into less flow accessible regions of the reservoir. Polymer producing microbes were used for their ability to produce abundant amount of polymer as verified in our laboratory tests. Polymer-producing bacteria identified in this study were tested for their ability to reduce the permeability of porous media. The ability of the polymer-producing bacteria to block

fluid flow and cause fluid diversion was also tested using coreflood experiments. The other main purpose was to mobilize oil by decreasing surface tension at the oil-water interface. Surfactant producing microbes were tested in coreflood experiments for their ability to mobilize oil. These experiments were conducted at the Petroleum Engineering Department at Texas A\&M University. Dr. Bryant facilitated oil samples and the original microbial samples.

The experimental set-up is shown in Figure 1. Blocks of Berea sandstone were obtained and cut in cylindrical shape, $25 \mathrm{~cm}$ in length and $2.5 \mathrm{~cm}$ in diameter. The cores were encased in rubber sleeves and placed inside Hassler coreholders. Coreflood experiments were conducted to determine permeability reduction due to in situ biopolymer production. Berea cores were injected with the appropriate culture-growing medium. Microbes were injected and left incubating for six days and permeability was determined. 
Coreflood experiments were also carried out to determine residual resistance factors $\left(\mathrm{F}_{\text {Ir }}\right)$. The aforementioned Berea cores were saturated with $0.5 \%$ brine solution and injected with 1 PV growth medium inoculated with NIPER 11. The cores were shut in for six days then flooded with brine, and flow rates and pressures were measured. The residual resistance factors $\left(\mathrm{F}_{\mathrm{rr}}\right)$ were then calculated using the following equation,

$$
\left.\mathrm{F}_{\mathrm{rr}}=\left(\mathrm{Q}_{\mathrm{w}} / \Delta \mathrm{P}\right)_{\text {brine }} / \mathrm{Q}_{\mathrm{w}} / \Delta \mathrm{P}\right)_{\text {AfterMicrobiallnjection }} \quad \text { (1), here } \mathrm{Q}_{\mathrm{w}} \text { is }
$$

the flow rate, and $\Delta \mathrm{P}$ is the pressure difference between fluid input and output.

Recovery experiments were carried out using microbial cultures and different nutrient media. After been placed in Hassler coreholders the Berea sandstone cores were evacuated and flushed with a $2 \%$ brine solution. Crude oil was injected into the cores until no additional water was produced, about 24 hours. Then, brine was injected until no more crude oil was produced. The core thus simulated a waterflooded ROS condition designated by $\mathrm{S}_{\text {orwf }}$.

Oil samples were obtained from the Delaware-Childress field in northeastern Oklahoma and from the Wilmington field in California. Delaware-Childress oil has a gravity of $31{ }^{\circ}$ API $(0.87 \mathrm{~g} / \mathrm{cm} 3)$, and Wilmington $17{ }^{\circ}$ API $(0.97 \mathrm{~g} / \mathrm{cm} 3)$. The first qualifies as a light/medium crude while the second was used as representative of heavy oils.

The following experimental procedure was adopted, the Berea sandstone cores that have been waterflooded to the residual saturation $S_{\text {orwf, }}$ are prepared for microbial injection. The pore volume of these cores varied from 25 to $30 \mathrm{ml}$. The cores were injected with $0.2 \mathrm{PV}$ of a bacterial cells solution (at a concentration of about $10^{6}$ cells/ml) in $1 \%$ peptone solution and $0.3 \mathrm{PV}$ of $10 \%$ sugar solution. Glucose, molasses and sucrose solutions were used as sugars. The cores were allowed to incubate at $33{ }^{\circ} \mathrm{C}$ for 1 week. The cores are flooded with brine at a rate of 0.3 to $0.6 \mathrm{~m} / \mathrm{day}$, and fractions of the core effluents were collected. The amount of oil in each fraction was determined, and the residual oil in the core, $\mathrm{S}_{\text {orcf, }}$ was calculated. The oil recovery efficiency $\left(E_{r}\right)$ was calculated using the following equation,

$$
E_{\mathrm{r}}=\left(\mathrm{S}_{\text {orwf }}-\mathrm{S}_{\text {orf }}\right) / \mathrm{S}_{\text {orwf }}
$$

Effluent fluids were separated and the water phase was separated for further analysis. Surface tension, viscosity and colony count measurements were conducted on the 
separated water phase. The same procedure was followed for the NIPER 1A and the NIPER 11 cultures.

Some experiments were carried out using NIPER 1A cultures changing the nutrient formulations. These experiments involved replacement of the ammonium salts by sodium salts. More details are provided in the tasks description sections. Some recovery experiments were carried out after the end point of the previous brine sweep. The cores were injected with a $0.2 \mathrm{PV}$ of a nutrient solution without ammonium salts. The residual oil in the core was calculated. The cores were incubated at $33{ }^{\circ} \mathrm{C}$ for three days. Finally, the cores were flooded with brine at a rate of 0.3 to $0.6 \mathrm{~m} /$ day, and fractions of the core effluents were collected. The amount of oil in each fraction was determined, and the final residual oil in the core, $S_{\text {ormf, }}$ was calculated. The total oil recovery efficiency $\left(E_{r t}\right)$ was calculated using the following equation,

$$
E_{\text {rt }}=\left(S_{\text {orwf }}-S_{\text {ormf }}\right) / S_{\text {orwf }}
$$

Comments and more details are given in the tasks description part.

\section{SIMULATION SECTION}

\section{Simulator Background}

UTCHEM, a chemical simulator developed by researchers at the University of Texas

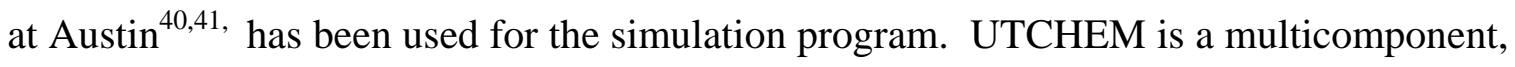
multiphase, three-dimensional compositional with variable temperature simulation model. The basic equations are as follows:

1) the mass balance equations, which are solved up to 21 species;

2) the aqueous phase pressure, which is obtained by an overall mass balance on volume occupying species (water, oil, surfactant, alcohol, and gas). The other phase pressures are computed by using the capillary pressures between phases;

3) the energy balance equation, which includes heat flow between the reservoir and the overburden rocks.

The flow equations allow for compressibility of rock and fluids, dispersion and molecular diffusion, chemical reactions, and phase behavior and are complemented by 
constitutive equations. The model includes options for multiple wells completed either horizontally or vertically. Aquifer boundaries are modeled as constant potential surfaces or as closed surfaces.

The flow equations are solved using a block-centered finite-difference scheme. The solution method is implicit in pressure and explicit in concentration (IMPES-like). Either one, two-point upstream, or third-order spatial, discretization is used. A brief description of the equations used in the code is provided below.

\section{Mass Conservation Equations}

The assumptions imposed when developing the flow equations are:

1) local thermodynamic equilibrium, except for tracers;

2) immobile solid phases;

3) slightly compressible rock and fluids;

4) Fickian dispersion;

5) ideal mixing; and

6) Darcy's law.

The boundary conditions are no flow and no dispersive flux across the impermeable boundaries.

The mass continuity for component $\mathrm{k}$ in association with Darcy's law is given in terms of overall volume of component $k$ per unit pore volume $\left(\widetilde{\mathrm{C}}_{\mathrm{k}}\right)$ as,

$$
\frac{\partial}{\partial \mathrm{t}}\left(\phi \widetilde{\mathrm{C}}_{\mathrm{k}} \rho_{\mathrm{k}}\right)+\underline{\nabla} \cdot \sum_{\mathrm{j}=1}^{\mathrm{n}_{\mathrm{p}}} \mathrm{r}_{\mathrm{k}}\left(\mathrm{C}_{\mathrm{kj}} \mathrm{u}_{\mathrm{j}}-\underline{\nabla} \bullet \underline{\underline{\mathrm{D}}}\right)=\mathrm{R}_{\mathrm{k}} \quad \text { (4), where the }
$$

overall volume of component $\mathrm{k}$ is the summation over all phases including the adsorbed phases,

$$
\tilde{\mathrm{C}}_{\mathrm{k}}=\left\{1-\sum_{\mathrm{k}=1}^{\mathrm{n}_{\mathrm{v}}} \mathrm{e}_{\mathrm{k}}\right\} \sum_{\mathrm{j}=1}^{\mathrm{n}_{\mathrm{p}}} \mathrm{S}_{\mathrm{j}} \mathrm{C}_{\mathrm{kj}}+\mathrm{e}_{\mathrm{k}} \text {, for } \mathrm{k}=1, \mathrm{n}_{\mathrm{cv}} \quad \text { (5), } \mathrm{n}_{\mathrm{cv}} \text { is the }
$$

total number of volume occupying components. These components are water, oil, surfactant, and gas, $n_{p}$ is the number of phases, $e_{k}$ is the adsorbed concentration of species $k$, and $\rho_{k}$ is the density of pure component $k$ at a reference phase pressure $P_{r}$ 
relative to its density at reference pressure $\mathrm{P}_{\text {ro, }}$, usually taken at a surface condition of 1 atm.

The phase flux from Darcy's law is given by,

$$
\underline{\mathrm{u}}_{\mathrm{j}}=-\frac{\mathrm{k}_{\mathrm{rj}}-\underline{\underline{\mathrm{k}}}}{\mu_{\mathrm{j}}} \bullet\left(\underline{\nabla} \mathrm{P}_{\mathrm{j}}-\gamma_{\mathrm{j}} \underline{\nabla h}\right) \quad \text { (6), where } \underline{\underline{k}}
$$

is the intrinsic permeability tensor and $h$ is the vertical coordinate, $k_{r j}, \mu_{j}$, and $\gamma_{j}$ are the relative permeability, viscosity, and specific weight for phase $j$. The source terms $R_{k}$ are a combination of all rate terms for a particular component.

\section{Pressure Conservation Equations}

The pressure equation is developed by summing the mass balance equations over all volume occupying components, substituting Darcy's law for the phase flux terms, using the definition of capillary pressure, and noting that $\left(\sum_{\mathrm{k}=1}^{\mathrm{n}_{\mathrm{cv}}} \mathrm{C}_{\mathrm{kj}}\right)=1$. The pressure equation in terms of the reference phase pressure (phase 1) is given by,

$$
\begin{aligned}
& \phi \mathrm{C}_{\mathrm{t}} \frac{\partial \mathrm{P}_{\mathrm{j}}}{\partial \mathrm{t}}+\underline{\nabla} \bullet \underline{\mathrm{k}} \bullet \lambda_{\mathrm{rTc}} \underline{\nabla} \mathrm{P}_{\mathrm{j}}=-\underline{\nabla} \bullet \sum_{\mathrm{j}=1}^{\mathrm{n}_{\mathrm{cv}}} \underline{\underline{\mathrm{k}}} \bullet \lambda_{\mathrm{rTc}} \underline{\nabla} \mathrm{h}+ \\
& +\underline{\nabla} \bullet \sum_{\mathrm{j}=1}^{\mathrm{n}_{\mathrm{cv}}} \underline{\underline{\mathrm{k}}} \lambda_{\mathrm{rTc}} \bullet \underline{\nabla} \mathrm{PC}_{\mathrm{jw}}+\sum_{\mathrm{j}=1}^{\mathrm{n}_{\mathrm{cv}}} \mathrm{Q}_{\mathrm{k}}
\end{aligned}
$$

(7), where the total relative mobility including the correction for fluid compressibility is given by $\lambda_{\text {rTc }}$ $=\sum_{\mathrm{k}=1}^{\mathrm{n}_{\mathrm{cv}}} \lambda_{\mathrm{rjc}}$, and $\lambda_{\mathrm{rjc}}=\frac{\mathrm{k}_{\mathrm{rj}}}{\mu_{\mathrm{j}}} \sum_{\mathrm{k}=1}^{\mathrm{n}_{\mathrm{cv}}} \mathrm{r}_{\mathrm{k}} \mathrm{C}_{\mathrm{kj}} . \mathrm{C}_{\mathrm{t}}$ is the total compressibility calculated as the volume weighted sum of matrix and component compressibilities.

\section{Fluid and Soil Properties}

Geological heterogeneities are the key factor that reduces the effectiveness of chemical enhanced recovery processes because their success depends on the delivery of injected chemicals and water into the subsurface. In order to capture some of the geological features, reservoir properties such as formation permeability, porosity, residual phase saturation, phase relative permeability, and phase capillary pressure are allowed to 
vary spatially in UTCHEM. Phase trapping functions and adsorption of both surfactant and polymer are modeled as a function of permeability.

\section{Polymer Adsorption}

Polymer adsorption can be an important mechanism for a chemical recovery project since it causes retardation polymer consumption. The retention of polymer and surfactant molecules in permeable media is due to both adsorption onto solid surfaces and trapping within small pores. UTCHEM uses a Langmuir-type isotherm to describe the adsorption level of a polymer or a surfactant, which takes into account the salinity, polymer concentration, and soil permeability. The adsorption is irreversible with concentration and reversible with salinity. The adsorbed concentration $\left(\Theta_{p}\right)$ is given by,

$$
e_{p}=\min \left\{\tilde{C}_{p}, \frac{a_{p}\left(\tilde{C}_{p}-e_{p}\right)}{1+b_{p}\left(\tilde{C}_{p}-e_{p}\right)}\right\}
$$

The minimum is taken to guarantee that the adsorption is no greater than the total polymer concentration. Adsorption increases linearly with effective salinity and decreases as follows,

$$
\mathrm{a}_{\mathrm{p}}=\left(\mathrm{a}_{\mathrm{p} 1}+\mathrm{a}_{\mathrm{p} 2} \mathrm{C}_{\mathrm{SEP}}\right) \mathrm{k}^{-0.5}
$$

The adsorption parameters $\mathrm{a}_{\mathrm{p} 1}, \mathrm{a}_{\mathrm{p} 2}$ and $\mathrm{b}_{\mathrm{p}}$ are found by matching laboratory polymer adsorption data. The effective salinity for polymer $\left(\mathrm{C}_{\mathrm{SEP}}\right)$ is,

$$
\mathrm{C}_{\mathrm{SEP}}=\frac{\mathrm{C}_{51}+\left(\beta_{\mathrm{p}}-1\right) \mathrm{C}_{61}}{\mathrm{C}_{\mathrm{w} 1}} \quad \text { (10), where }
$$
$\mathrm{C}_{51}, \mathrm{C}_{61}$, and $\mathrm{C}_{\mathrm{w} 1}$ are the anion, calcium, and water concentrations in the aqueous phase and $\beta_{p}$ is experimentally determined.

\section{Viscosity}

Liquid phase viscosities are modeled in terms of pure component viscosities and the phase concentrations of the organic, water and chemicals,

$$
\mu_{\mathrm{k}}=\mathrm{C}_{\mathrm{wk}} \mu_{\mathrm{k}} \mathrm{e}^{\alpha_{\mathrm{w}}\left(\mathrm{C}_{\mathrm{ok}}+\mathrm{C}_{\text {chk }}\right)}+\mathrm{C}_{\mathrm{ok}} \mu_{\mathrm{k}} \mathrm{e}^{\alpha_{\mathrm{o}}\left(\mathrm{C}_{\mathrm{wk}}+\mathrm{C}_{\text {chk }}\right)}+\mathrm{C}_{\text {chk }} \mu_{\mathrm{k}} \mathrm{e}^{\alpha_{\mathrm{ch}}\left(\mathrm{C}_{\mathrm{wk}}+\mathrm{C}_{\mathrm{ok}}\right)} \quad \text { (11), for } \mathrm{k}=
$$
water, oil or chemical. 
The $\alpha$ parameters are determined by matching laboratory microemulsion viscosities at several compositions. In the absence of polymer, water and oil phase viscosities are reduced to pure water and oil viscosities. When polymer is present $\mu_{\mathrm{w}}$ is replaced by $\mu_{\mathrm{p}}$ defined below.

The viscosity of the polymer solution depends on the concentration of polymer and on salinity,

$$
\mu_{\mathrm{p}}^{0}=\mu_{\mathrm{w}}\left\{1+\left(\mathrm{A}_{\mathrm{pw}} \mathrm{C}_{\mathrm{pw}}+\mathrm{A}_{\mathrm{po}} \mathrm{C}_{\mathrm{pw}}^{2}+\mathrm{A}_{\mathrm{pch}} \mathrm{C}_{\mathrm{pw}}^{3}\right) \mathrm{C}_{\mathrm{SEP}}^{\mathrm{Sp}}\right\} \quad \text { (12), where }
$$

$\mathrm{C}_{\mathrm{pw}}$ is the polymer concentration in water, $\mu_{w}$ is the water viscosity, $\mathrm{A}_{\mathrm{pi}}$ are constants. The factor $C_{S E P}^{S p}$ allows for dependence of polymer viscosity on salinity and hardness.

The reduction in the viscosity of the polymer solution is a function of shear rate $(\gamma)$ and is modeled by using Meter's equation,

$$
\mu_{\mathrm{p}}=\mu_{\mathrm{w}}+\left(\mu_{\mathrm{p}}^{\mathrm{o}}-\mu_{\mathrm{w}}\right) /\left(1+\left\{\gamma / \gamma_{1 / 2}\right\}^{\mathrm{P \alpha}-1}\right) \quad \text { (13), where }
$$

$\gamma_{1 / 2}$ is the shear rate at which viscosity is the average of $\mu_{\mathrm{p}}^{0}$ and $\mu_{w}$, and $\mathrm{P}_{\alpha}$ is an empirical coefficient.

\section{Microbial Enhanced Oil Recovery Model}

Microbial enhanced oil recovery (MEOR) process is based on the injection of large quantities of nutrients in the reservoir. These nutrients accelerate the growth of both the injected cultures and the indigenous microorganisms. During growth of bacteria, several metabolites such as alcohols, surfactants, and polymers are produced. Among these bioproducts, surfactants, biomass, and polymers are potentially the most useful products for improved oil recovery. The implementation includes very general kinetics and mass transfer models so it can accommodate any number of substrates, electron acceptors, and biological species. It can model the substrate inhibition, substrate competition, electron acceptor inhibition, abiotic decay, biomass growth, and biomass attachment. The model also includes permeability reduction due to biomass growth and retention. The formation of products such as bio-surfactants and bio-polymers has been incorporated.

In the simplest case of a single subtrate and a single biological species it is generally accepted that the mass of new cells (X) synthesized per unit mass of substrate (S) removed is constant for a given substrate and bacterial species, 


$$
\frac{d S}{d t}=\frac{\mu_{\max } S X}{Y\left(K_{s}+S\right)}
$$

The term $\frac{\mu_{\max }}{Y}$ can be replaced by the term $\mathrm{k}$, which is referred to as the degradation rate constant or maximum rate of substrate removal per unit weight of biomass. This results in the following expression for the rate substrate degradation,

$$
\frac{d S}{d t}=\frac{k S X}{K_{s}+S}
$$

Similarly an expression for the rate of biomass formation is,

$$
\frac{d X}{d t}=\frac{Y k S X}{K_{s}+S}-b X
$$

$\mu_{\max }$ and $\mathrm{K}_{\mathrm{s}}$ are the Monod kinetic parameters, $\mathrm{b}=$ endogenous decay constant, $\mathrm{Y}$ is the yield coefficient, cell mass produced per mass of substrate biodegraded.

Similar equations can be written for several substrates, electron acceptors; attached, and non-attached biomass.

If significant biomass growth occurs in the modeling domain, then the reservoir porosity will be reduced, with a concomitant reduction in reservoir permeability. Because biological growth is limited to the aqueous phase, porosity reduction occurs only at the expense of the aqueous phase. Permeability in the flow direction is calculated based upon the Carman-Kozeny ${ }^{40}$ equation:

$$
\mathrm{k}_{\mathrm{x}}=\mathrm{d}_{\mathrm{p}}^{2} \phi^{3} / 300(1-\phi)^{2}
$$

is the permeability in the flow direction $(\mathrm{cm})$, and $\mathrm{d}_{\mathrm{p}}$ is the particle diameter $(\mathrm{cm})$. The $\mathrm{y}$ and z permeability values are adjusted using equation (17).

\section{Surfactant/Brine/Oil Phase Behavior}

The surfactant-oil-water phase can be represented as a function of effective salinity once the binodal curve and the tie-lines are described. The phase behavior model in the UTCHEM simulator uses Hand's rule, and is based on the work by Nelson and Pope ${ }^{42}$, among others. The effective salinity increases with the divalent cations bound to micelles (Hirasaki ${ }^{43}$ ) and decreases as the temperature increases for anionic surfactants. 
The formulation of the binodal curve using Hand's rule is assumed to be the same in all phase environments. Hand's rule is based on the empirical observation that equilibrium phase concentration ratios are straight lines on a log-log plot scale. The binodal curve is computed from,

$$
\frac{C_{3 j}}{C_{2 j}}=A\left\{\frac{C_{3 j}}{C_{1 j}}\right\}^{B} \text {, with } j=1,2 \text {, or } 3 \quad \text { (18), where } A
$$

and $\mathrm{B}$ are empirical parameters. For a symmetric binodal curve where $\mathrm{B}=-1$ all phase concentrations are calculated explicitly in terms of oil concentration, $\mathrm{C}_{2 \mathrm{j}}$.

$$
C_{3 j}=0.5\left[-\mathrm{A} \mathrm{C}_{2 j}+\sqrt{\left(\mathrm{A} \mathrm{C}_{2 \mathrm{j}}\right)^{2}+4 \mathrm{~A}\left(1-\mathrm{C}_{2 \mathrm{j}}\right)}\right] \text {, for } \mathrm{j}=1,2 \text {, or } 3
$$

\section{TASKS DESCRIPTION}

\section{Task 1 - Characterization of Surfactant and Polymer-Producing Bacteria}

\section{Subtask 1.1 - General Bacterial Physiology}

The microorganisms selected for the microbial surfactant-polymer system were characterized to determine how relevant physical and chemical factors affect their growth. Growth experiments were conducted to determine optimum growth conditions. The original microbial samples were provided by Dr. R. Bryant and grown in a Thyoglycolate broth medium at ACORN Biotechnical, a private biotechnology company. Surface tension and viscosity for NIPER 1A and NIPER 11A samples were recorded for more than 10 consecutive months. Periodic plate counts were also measured in order to determine microbial numbers. Figure 2 shows results for NIPER 1A samples grown in both broth media. The microbial population is very resilient and remained viable for a long period of time when provided with adequate nutrients. The microbes grown in trypticase soy broth grew more than those grew on Brewer thioglycolate broth. These results are typical of all our experiments. Probably the fact that the trypticase culture was grown and maintain under anaerobic conditions influenced the results.

Typical results corresponding to our laboratory experiments are shown in Table 2. We can see that NIPER 1A cultures produced a significant reduction in the medium surface tension. This behavior was observed for all the NIPER 1A cultures grown independently of the sugar used. In general sucrose and molasses cultures produced 
similar results while the values measured using glucose cultures were lower. The same trends were observed for standard plate counts (cfu/ml) and viscosity measurements. These results verified that NIPER $1 \mathrm{~A}$ is a good surfactant producer and that there is a direct relationship between population size and surfactant production. The bigger the population the bigger the amount of biosurfactant produced and, therefore, the lower the surface tension is. NIPER 11 results showed a much smaller reduction in surface tension even when the population growth of these microbes was about the same size of NIPER 1A populations. This result reflects the fact that NIPER 11 is a poor surfactant producer, and no significant change has resulted from the use of different sugars as nutrients. Our results confirmed than NIPER 11 is a good polymer producer, but it is not a good surfactant producer.

In order to study the influence of different sugars on the growth rate $1 \mathrm{ml}$ of the broth inoculum was placed in $250 \mathrm{ml}$ flasks containing $150 \mathrm{ml}$ of modified medium E. The modification involved adding $0.1 \%$ yeast extract and replacing the carbon source with glucose, sucrose, fructose and molasses. Dr. Bryant provided the molasses used in these experiments. They were originally obtained from Pacific Molasses Co. at Oklahoma city, and its composition is as follows: mineral content: total ash $8.1 \%$, calcium: $0.8 \%$, phosphorous: $0.08 \%$, magnesium: $0.35 \%$, potassium: $2.4 \%$, sulfur: $0.8 \%$, and sodium: $0.2 \%$. The amount of total suspended solids is $74 \%$, of which $3 \%$ is total protein, $48 \%$ is total sugar (sucrose), and the remaining $23 \%$ is fiber. The concentration of molasses was $4 \%$ by weight in tap water.

Figure 3 shows a typical growth curve for the different carbon sources used in this study. The growth curve depicts all the classical kinetic periods. At first, the number of microorganisms did not show appreciable growth (lag period). After this lag period the microbial population growths exponentially until reaches equilibrium and after approximately four days started to decline in numbers. The same behavior was appreciated for all our experiments in different growth media. Similar results were obtained for the NIPER 11 samples.

NIPER 1A samples grown using glucose as carbon source grew well, albeit at smaller rate, than the ones grown using pure sucrose or molasses as carbon sources. This was a surprise because Javaheri et al. ${ }^{28}$ have reported glucose as the preferred carbon source for 
growth of Bacillus licheniformis strain JF-2 (ATCC 39307). However, Ramsay et al. ${ }^{31}$ reported slower growth for Bacillus licheniformis strain NRC 9012 using glucose or a glucose-fructose mixture than using sucrose as carbon source.

\section{Subtask 1.2 - Surfactant Production}

Biosurfactants cause a decrease in the surface tension between air and culture phases. Biosurfactants also affect the interfacial tension between the aqueous, and oil phase, which is the primary mechanism by which surfactant-producing bacteria mobilize oil. Selected strains that produce large amounts of biosurfactants were tested for their effects on interfacial tension between aqueous and oil phases. Surface tension was measured at various stages of culture growth and over a reasonable range of nutrient levels using the $d u$ Nuoy ring method. Some measurements of solution-oil interfacial tension were done using the spinning drop method at the Petroleum Engineering Department at TAMU.

Figure 4 depicts typical surface tension values for cultures grown using medium $\mathrm{E}$ and different carbon sources. These results correspond to the cultures depicted in Figure 3. Surfactant production was directly proportional to cell growth; as the cell density increased the surface tension decreased. The greatest decreased in surface tension was observed during the log phase (36 hours). Figures 3 and 4 prove that 84 hours is enough time for surfactant production in flasks experiments.

NIPER 1A samples grown using glucose as carbon source produced very small decrease in the measured surface tension, 5 to $10 \mathrm{mN} / \mathrm{m}$. This effect cannot be explained on the basis of microbial population size only. Bryant and Douglas ${ }^{35}$ have reported similar behavior for NIPER 1A cultures. Lin et al. ${ }^{33}$ reported the opposite behavior in their detailed analysis of the metabolic conditions for biosurfactant production using Bacillus licheniformis JF-2 cultures. Lin et al. ${ }^{33}$ reported the maximum amount of biosurfactant being produced by a culture that used glucose as the sole carbon source. There was not appreciable difference between the cultures grown using pure sucrose and molasses as carbon sources.

The surfactant produced by NIPER $1 \mathrm{~A}$ is most likely a lipopeptide similar to surfactin produced by Bacillus subtilis ${ }^{23}$. Cultures of Bacillus licheniformis JF-2 also produce a 
biosurfactant lipopeptide, consisting of a C15 fatty acid tail linked to a peptide moiety very similar to surfactin ${ }^{33}$.

These results verified that NIPER $1 \mathrm{~A}$ is a good surfactant producer and that there is a direct relationship between population size and surfactant production. NIPER 11 results showed a much smaller reduction in surface tension even when the population growth of these microbes was about the same size of NIPER 1A populations. This result reflects the fact that NIPER 11 is a poor surfactant producer and no significant change has resulted from the use of different sugars as nutrients.

Figure 5 depicts the interfacial tension (IFT) between a NIPER 1A culture, and an oil phase (n-decane). The interfacial tension decreases continuously for the first 24 hours. The IFT value becomes constant for longer periods of time. These data correspond to a sucrose modified culture medium $\mathrm{E}$ with $0.1 \%$ yeast extract added.

The influence of brine concentration on interfacial tension for several oils was also studied. Toluene, n-hexane and n-decane were used as representative oil phases. It has been reported that NIPER 1A can produce biosurfactants up to $10 \mathrm{wt} \%$ brine content, but the optimum values are produced by $4-5 \mathrm{wt} \%$ solutions. Excess of brine has been reported as leading to suppression of biosurfactant production ${ }^{33}$. Figure 6 shows smaller IFT values for hydrocarbon with high carbon number (n-decane) while aromatics (toluene) produced the smallest measured IFTs. A $5 \mathrm{wt} \%$ brine concentration produced the best IFT values. In this work we did not determine the optimal salinity concentration. The optimal salinity is the concentration value that produces three faces in the microbial culture/oil/biosurfactant system ${ }^{40}$. This condition is considered the most favorable for oil recovery because produces the maximum oil solubility and the minimum interfacial tension among all the phases present. This phase behavior receives the name of Winsor type III or just type III.

The salinity value determines two other possible phase behaviors involving two phases present. At low salinity below critical micelle concentration (CMC), the two phases are an aqueous phase; containing all the surfactant, electrolytes, and dissolved oil; and an excess oil phase. At values above the CMC, a microemulsion, and a mostly pure oil phase are present. This type of phase behavior is called Winsor type I, or alternatively type II(-). For high salinity, an excess water phase and a microemulsion phase containing 
most of the surfactant and oil; and some solubilized water exist. This type of phase behavior is called Winsor type II, or alternatively type II(+). The phase diagram is also strongly influenced by the concentration of divalent ions $\left(\mathrm{Ca}^{2+}, \mathrm{Mg}^{2+}\right.$, etc.).

In this work we only observed type II(-) phase behavior, i.e., an aqueous phase; containing all the surfactant, electrolytes, and some dissolved oil; plus an excess oil phase.

\section{Subtask 1.3 - Polymer Production}

Polymer-producing bacteria polymerize carbohydrates present in growth substrate into extra-cellular polysaccharide biopolymers. The production of soluble biopolymers causes viscosity increases in the microbial growth medium. Polymer production can be stimulated by nutrient manipulations and/or other external factors. Characterization of polymer-producing microorganisms included a study of biopolymer formation, and viscosity increases. Optimum polymer production was determined by inspection, chemical assays which included alcohol precipitation, and by measuring viscosity increases with a viscometer.

Several experiments were conducted using a well-known polymer-producing microbe NIPER 11. Stepp et al. ${ }^{12}$ reported that NIPER 11 is a strain of Leuconotoc mesenteroides that ferments sucrose and produces lactic acid, acetic acid, ethanol and an insoluble dextran polymer. These polysaccharide polymers consist of mostly linear monosaccharides chains linked by $\alpha-1 \rightarrow 6$ bonds with generally short side branches. The microbe grows using a variety of carbon sources, but only produces the dextran polymer in the presence of sucrose ${ }^{30}$. This fact can be used to place the microbial population in depth inside the reservoir and later on metabolically activate the biopolymer formation by adding appropriate nutrients. Jenneman et al. ${ }^{30}$ studied the influence of nutrients on gel production. The authors concluded that growth of Leuconotoc mesenteroides in a synthetic sucrose-based medium resulted in production of rigid, bulk dextran gels, whereas, growth on a beet-molasses medium produced a loose, colloidal gel.

Inspection of essay tubes seeded with $1 \mathrm{ml}$ of NIPER 11 inoculum, and tryptic soy broth growth medium (Table 1) produced copious amounts of biopolymer. A white paste appears in the bottom of the essay tube within a few days from inoculation. The viscosity 
of NIPER 11 cultures did not significantly increase though. This fact indicates that the produced biopolymer is largely insoluble in the culture medium and; therefore, does not increase the aqueous medium viscosity. This result agrees with the findings of Jenneman et al. ${ }^{30}$.

Several Bacillus licheniformis strains produce biopolymers. Stepp et al. ${ }^{12}$ studied a strain, designated as $\mathrm{TG}_{2}$-32, for possible use to improve conformance control. This microorganism has been isolated from arid conditions in Arizona. This bacteria produced a levan polymer similar to the one reported by Ramsay et al. ${ }^{31}$. These polymers are $\beta$-DFructofurannans with linear chains of approximate 12 units and $\beta-2 \rightarrow 6$ monosaccharide bonds. Stepp et al. ${ }^{12}$ results support $\mathrm{TG}_{2}$-32 use as a permeability reducing agent. However, NIPER 11 was found to be more effective in this role.

Ramsay et al. ${ }^{31}$ used a Bacillus licheniformis strain, NRC 9012, to produce a waterinsoluble, metabolically controlled, levan polymer. The microbial strain grows with several carbon sources, but only produces polymer when fermenting sucrose in presence of nitrate salts as nitrogen source. The authors concluded that the Bacillus culture or a preparation of its extracellular enzymes might be used as a selective plugging agent in undesirable, high permeability zones in oil reservoirs where the temperature is less than $50{ }^{\circ} \mathrm{C}$, the $\mathrm{pH}$ is between 6 and 9 , the salt concentration is less than $4 \%$, and the pressure less than 500 atm.

Several studies were carried out in order to determine the possibility of producing polymers using NIPER 1A. These studies involved the used of the modified medium E, ATCC 1502, described in Table 1. This medium without carbon and nitrogen sources will inhibit growth, and shut-off surfactant production. The addition of sucrose as carbon source and sodium nitrate as nitrogen source will trigger polymer production. Methanol precipitation tests have been used to determine the polymer production. The polymer production has also been tested in coreflood experiments. These experiments involved accurate nutrient manipulation; therefore, are described in more detail in the next two sections.

\section{Task 2 -Evaluation of Nutrient Regimes}

\section{Subtask 2.1 - Polymer Stimulation}


Biopolymers of interest for profile modification in MEOR applications have polysaccharide (carbohydrate) backbones. Many of the biopolymers produced by bacteria are glucans made of repeating glucose residues. Different linkages between the glucose residues and functional group substitutions on the glucose monomer give the different polymers their distinct properties. Nonetheless, they are all carbohydrates and bacteria need nutrients rich in carbohydrates to produce the polymers. In addition, some bacteria have additional nutrient requirements such as amino acids and vitamins. For a microbial polymer system to be economically attractive for MEOR applications, it needs a low-cost nutrient package that is rich in carbohydrates and any other essential nutrients.

A valuable feature of the microbial polymer systems investigated in this project is that the microbial cells can be grown without producing polymer, and then stimulated by nutrient manipulation to begin producing polymer. The advantage of this feature for MEOR is that cells can be injected deeply into the reservoir and then stimulated by nutrient injections to produce biopolymer in-situ. An important aspect of this research was to investigate the use of nutrient systems to control and stimulate polymer production. Different nutrient systems, including low-cost industrial grade products, were examined to determine efficacy for stimulating polymer production.

Studies were carried out in order to determine the possibility of producing polymers using NIPER 1A. These studies involved the used of the modified medium E, ATCC 1502, described in Table 1. This medium without carbon and nitrogen sources will inhibit growth, and shut-off surfactant production. NIPER $1 \mathrm{~A}$ is a spore-forming microbe that in situations of nutrient depravation will form spores. These spores are 0.2$0.5 \mu \mathrm{m}$ in diameter and 1-2 $\mu \mathrm{m}$ in length. These spores can be injected without problem inside reservoirs with permeability as low as $100 \mathrm{mDarcies}$. Appropriate nutrients can be injected after microbial placement in order to activate cell growth and chemicals production $^{13,44}$.

After several laboratory trials we found that adding $10 \mathrm{~g} / \mathrm{l}$ of sucrose as carbon source and $5 \mathrm{~g} / \mathrm{l}$ of $\mathrm{NaNO}_{3}$ as nitrate source to our modified medium $\mathrm{E}$ will produce a paste-like white precipitate after methanol treatment. A $1 \mathrm{ml}$ NIPER 1A inoculum was injected into a $10 \mathrm{ml}$ essay tube filled with the modified medium plus the carbon and nitrogen source. The tube was incubated at $33{ }^{\circ} \mathrm{C}$ for three days in an oven. The cells were removed by 
centrifugation, methanol was added up to $3 \mathrm{~V}$, and the supernatant recentrifuged at 25,000 g for $15 \mathrm{~min}$. The top portion of the solution was clear with increasing opacity towards the bottom of the tube where some solid had accumulated. The amount of methanol was calculated depending upon previous experiments results. If there was no previous success the amount of methanol was increased until the appearance of a precipitate.

In similar experiments using NIPER 11 the amount of precipitate was significantly higher than in the NIPER 1A cases. In most cases a precipitate was observed directly in the culture before centrifugation and methanol addition.

The surface tension and viscosity of the bacterial solution were determined before and after the precipitation experiments. These results allowed us to determine whether the precipitate contained some biosurfactant. Determination of the polymer solubility was done based upon the viscosity measurements. Soluble biopolymers, such as xanthams, will increase the viscosity of the culture while insoluble ones such as some dextrans and levans will not.

Table 2 shows typical viscosities and surface tension of our chemical experiments. NIPER 1A cultures showed very little decrease in surface tension suggesting that the precipitate is mostly a polymer and not a surfactant. There was very little viscosity increase suggesting that the polymer is mostly insoluble. This polymer is most likely a levan as reported by Stepp et al. ${ }^{12}$, and Ramsay et $\mathrm{al}^{31}$ for other Bacillus licheniformis strains.

In conclusion our chemical experiments point to the production of a water-insoluble biopolymer by feeding starving NIPER 1A cells with our modified medium E.

In order to study the influence of cheap nutrients on the biopolymer sucrose was replace by molasses $(20 \mathrm{~g} / \mathrm{l})$ in some experiments. The results were very similar to the case were sucrose was used as carbon source. Visual observation would suggest that even more polymer was produced using molasses instead of sucrose. This result suggests that commercial molasses are an economic alternative to the use of sucrose. 


\section{Task 3 -Testing of Microbial Systems in Porous Media}

An important part of this research was to validate the concept that a microbial surfactant-polymer system can be designed to advance current technology for improving oil recovery from porous media in the laboratory. We did this in a series of experiments designed to show advantages of concerted microbial polymer system blockage of watered out high permeability zones and microbial surfactant-improved mobilization of oil from oil-bearing zones. The two mechanisms for oil recovery that were developed in this research program, increased oil mobility and profile modification, were tested individually in coreflood experiments. Recovery and permeability reduction experiments were performed in order to study the performance of the microbial systems. The processes were then combined in more complex experiments to test the performance of the combined system.

\section{Subtask 3.1 - Microbial Transport}

Before any MEOR technique, such as in-situ surfactant and gas production or flow diversion, can be realized, a basic understanding of how bacteria are transported through porous media and how their retention affects the permeability of the media is needed. Bacterial transport through, and retention by, porous media differ from particle transport because cells increase in number and can produce polysaccharides, which affect their ability to adhere to surfaces.

Several research efforts point to the need of studying very careful the placement of microbial systems for plugging high permeability areas ${ }^{45,46,47}$. For plugging higherpermeability strata containing larger pore throats, Jack et al. $^{45}$ demonstrated the importance of using live, polymer-producing bacteria (e.g., biofilms) vs. dead, metabolically inactive bacteria (i.e., particulates). Lappan and Fogler ${ }^{46}$ concluded that insoluble polymers (i.e., dextran) produced by the bacterium Leuconostoc mesenteroides, are largely responsible for losses in permeability they observed in high-permeability ceramic cores. However, Jack et al. $^{45}$ indicated that exopolymer-producing bacteria are not ideally suited for injection into a porous matrix since they are highly retained at the inlet face. Subsequently, Thompson and $\mathrm{Jack}^{47}$ patented a novel method for injecting $L$. mesenteroides whereby the bacterial inoculum was grown in a nutrient medium 
containing glucose that did not stimulate exopolymer production and then, fed a sucrosecontaining medium (e.g., molasses) to trigger exopolymer production in-situ.

Experiments were conducted in Berea sandstone cores to study microbial transport in porous media. NIPER 11 and NIPER 1A cultures were used. The microbes shape and size have significant influence on their transport properties. NIPER 11 is a small spherical strain of Leuconostoc Mesenteroides, while NIPER 1A is a rod-shaped strain of Bacillus licheniformis.

A modification of the set-up depicted in Figure 1 was used. Berea sandstone cores of $2.5 \mathrm{~cm}$ diameter and $25 \mathrm{~cm}$ lehgth were used. The Berea cores were placed in Hasslertype core holders following the procedure already described in the experimental section. NIPER 11 cultures were grown in tryptic soy broth medium and NIPER 1A in medium E plus $0.1 \mathrm{wt} \%$ yeast extract added. An inoculum was prepared by centrifugation at 14,000 g for 20 minutes. The bottom, containing the live cells, was separated washed and added to the medium to be used in injection and incubated at $33{ }^{\circ} \mathrm{C}$ for 72 hours.

A $1 \mathrm{ml}$ inoculum of NIPER 11 was injected into 1\% peptone solution where $10 \mathrm{~g} / \mathrm{l}$ of glucose as carbon source was added. This medium promotes growth, but not polymer production. The inoculum concentration was determined by colony counting as $210^{8}$ cells/ml. The cores were injected with $3 \mathrm{PV}$ of inoculum at $20 \mathrm{ft} / \mathrm{day}$, followed by $8 \mathrm{PV}$ of sterile brine at $20 \mathrm{ft} /$ day. No increase in pressure was observed during the corefloods. Figure 7 shows typical results. It was found that retention of NIPER 11 in the Berea core is very high. This result agrees with the findings of Parli et al. ${ }^{48}$. In the case of NIPER $1 \mathrm{~A}$ the inoculum concentration by colony counting was found to be $1.3510^{8}$ cells $/ \mathrm{ml}$. The cores were injected with 1.2 PV of inoculum at $20 \mathrm{ft} /$ day, followed by $8 \mathrm{PV}$ of sterile brine at $20 \mathrm{ft} /$ day. No increase in pressure was observed during the corefloods. Typical results are shown in Figure 8. The transport rate of NIPER 1A inside the Berea core was very low, even lower that the transport rate of NIPER 11 cultures.

All the injections were of inoculum in a growth-promoting nutrient system. Parli et al. ${ }^{48}$ indicated that transport efficiency increases when cells are injected in a nutrient system. The nutrient system may lessen the electrostatic and hydrophobic effects of the cell, or it may compete for adsorption sites on the porous medium, thus allowing a greater number of cells to transport through the core ${ }^{48}$. 
These results point to the need to find better procedures for microbial placement. In the case of NIPER 1A we can take advantage of the fact that the microbe in situations of nutrient depravation will form spores. The spores are $0.2-0.5 \mu \mathrm{m}$ in diameter and 1-2 $\mu \mathrm{m}$ in length, and can be injected without problem inside reservoirs with permeability as low as 100 mDarcies. Appropriate nutrients can be injected after microbial placement in order to activate cell growth and chemicals production ${ }^{13,44}$.

\section{Subtask 3.2 - Improved Oil Mobility}

The microbial surfactant system produces biosurfactants, which mobilize crude oil by lowering the interfacial tension between aqueous and non-aqueous phases. In addition, microorganisms produce a variety of primary metabolites such as, alcohols, organic acids, and carbon dioxide, which can act synergistically with the surfactants by further reducing interfacial tension and reducing oil viscosity. A series of coreflood experiments were designed to test the microbial surfactant system for mobilizing residual crude oil in porous media.

Recovery experiments were carried out using NIPER 1A and NIPER 11 cultures. The experimental set-up is a modification of the one shown in Figure 1. Blocks of Berea sandstone were obtained and cut in cylindrical shape, $25 \mathrm{~cm}$ in length and $2.5 \mathrm{~cm}$ in diameter. The cores were encased in rubber sleeves and placed inside in Hassler coreholders. Then, the Berea sandstone cores were evacuated and flushed with a brine $2 \%$ solution. Crude oil was injected into the cores until no additional water was produced, about 24 hours. Then, brine was injected until no more crude oil was produced. The core thus simulated a waterflooded ROS condition designated by $\mathrm{S}_{\text {orwf }}$.

Oil samples were obtained from the Delaware-Childress field in northeastern Oklahoma and from the Wilmington field in California. Delaware-Childress oil has a gravity of $31{ }^{\circ}$ API $(0.87 \mathrm{~g} / \mathrm{cm} 3)$, and Wilmington $17{ }^{\circ}$ API $(0.97 \mathrm{~g} / \mathrm{cm} 3)$. The first qualifies as a light/medium crude while the second was used as representative of heavy oils.

The following experimental procedure was adopted. The Berea sandstone cores that have been waterflooded to the residual saturation $S_{\text {orwf, }}$ are prepared for microbial injection. The pore volume of these cores varied from 25 to $30 \mathrm{ml}$. The cores were 
injected with $0.2 \mathrm{PV}$ of a bacterial cells solution (at a concentration of about $10^{6}$ cells $/ \mathrm{ml}$ ) in $1 \%$ peptone solution and $0.3 \mathrm{PV}$ of medium $\mathrm{E}$ with $10 \%$ sugar solution and $0.1 \%$ yeast extract. Glucose, molasses and sucrose solutions were used as sugars. The cores were allowed to incubate at $33{ }^{\circ} \mathrm{C}$ for 1 week. The cores are flooded with brine at a rate of 0.3 to $0.6 \mathrm{~m} /$ day, and fractions of the core effluents were collected. The amount of oil in each fraction was determined, and the residual oil in the core, $\mathrm{S}_{\text {orcf, }}$, was calculated. The oil recovery efficiency $\left(\mathrm{E}_{\mathrm{r}}\right)$ was calculated using the following equation,

$$
\mathrm{E}_{\mathrm{r}}=\left(\mathrm{S}_{\text {orwf }}-\mathrm{S}_{\text {orcf }}\right) / \mathrm{S}_{\text {orwf }}
$$

Effluent fluids were separated and the water phase was separated for further analysis. Surface tension, viscosity and colony count measurements were conducted on the separated water phase. The same procedure was followed for the NIPER $1 \mathrm{~A}$ and the NIPER 11 cultures.

Table 3 shows typical results of our recovery experiments using the two types of oils and cores of different absolute permeability. NIPER 1A populations produced a significant decrease in the solutions surface tensions while NIPER 11 cultures only slightly change the surface tension of the produced solutions. NIPER 11 cultures increase slightly the viscosity of the solutions while NIPER 1A cultures did not do so. Similarly to the laboratory measurements no significant differences in population values were observed between the two kinds of microbes using the same sugars as nutrients. Sucrose and molasses performed better as nutrient than glucose, but acceptable values were measured for the glucose solutions. NIPER 1A solutions showed better recovery efficiency $\left(E_{r}\right)$ than the NIPER 11 ones.

No significant difference in permeability reduction was observed between experiments conducted using low permeability and medium permeability cores. Slightly better recovery was measured using medium permeability cores than low permeability cores.

Oil recovery results are shown in Figures 9 and 10. The results in Figure 9 were obtained using NIPER 1A samples while the results shown in Figure 10 were measured using NIPER 11 samples. Both microbial samples show increase recovery as the injected brine volume increases compared to the residual oil saturation after waterflooding. Residual recovery efficiency $\left(E_{r}\right)$ in the NIPER 1 A cultures was above $53 \%$ (Table 2). 
NIPER 11 cultures showed lower, but still respectable recovery values. Recovery values for molasses nutrient solutions were only slightly smaller than the sucrose values in the NIPER 1A experiments and practically the same in the NIPER 11 experiments. The recovery curves are very similar, but the main mechanism of oil recovery in the case of NIPER 1A is interfacial tension reduction while in the case of NIPER 11A is improved areal sweep efficiency. NIPER 1A samples are well-known producers of surfactant while NIPER 11A cultures produce abundant amounts of dextran biopolymers.

Similar residual oil saturation curves were obtained when heavy oil samples were used (Chaffee). The residual recovery efficiency was higher in this case for NIPER 1A samples, 72.2\%, than in the experiments done using NIPER 11, 47.1\%. A possible explanation could be the higher permeability of the core used in the experiments with NIPER 11 cultures. The NIPER 11 microbes could only partially shut-off the high permeability pores, therefore, a significant amount of by-pass led to lower recovery efficiency. These results are similar to the ones reported by Bryant and Douglas ${ }^{35}$.

More complex recovery experiments were carried out to study possible nutrient manipulation in order to produce a biopolymer using NIPER 1A cultures. The cores were injected with $0.2 \mathrm{PV}$ of a bacterial cells solution (at a concentration of about $10^{6}$ cells $/ \mathrm{ml}$ ) in $1 \%$ peptone solution and $0.3 \mathrm{PV}$ of medium $\mathrm{E}$ with $1 \%$ sucrose solution and $0.5 \% \mathrm{NaNO}_{3}$ without yeast extract. The cores were allowed to incubate at $33{ }^{\circ} \mathrm{C}$ for 1 week. The experiments were carried out following the procedure described above. Typical results are presented in Figure 11. In this case there is less recovery than in the surfactant-producing mode. The surface tension of the water effluent is very close to water without any surfactant. The interfacial tension between the recovered oil and the water solution is also close to the value in absence of surfactants, $8 \mathrm{mN} / \mathrm{m}$. The recovery for NIPER 1A cultures using the modified growth medium is better than equivalent values measured using NIPER 11, Figure 10. We can speculate that there is some surfactant production that helps to mobilize the oil followed by biopolymer production that contributes to conformance control. The combination of these two processes produces better recovery than the conformance control alone produced by NIPER 11 .

Some experiments were carried out taking the core after injection of 3.3 PV, and injecting 0.3 PV of the modified medium $\mathrm{E}$ including $\mathrm{NaNO}_{3}$ as nitrogen source. After 
the new nutrient injection more brine was added. Typical results of these experiments are shown in Figure 12. The extra injection of nutrients seems to increase recovery even when the increase is modest. The change in nutrients can produce in-situ some polymer that helps to mobilized the oil 'turned loose' by the previous surfactant production.

\section{Subtask 3.3 - Improved Sweep Efficiency}

The purpose of the microbial polymer systems for improving oil recovery is to block fluid flow through watered out thief zones in the reservoir and divert fluid flow into less accessible regions of the reservoir. Polymer-producing bacteria identified in this study were tested in coreflood experiments for their ability to reduce the permeability of porous media. These tests were designed to test performance and limitations of the novel microbial polymer system.

Coreflood experiments were conducted to determine permeability reduction due to in situ biopolymer production. The experimental set-up is shown in Figure 1. Berea cores were injected with the medium E growing medium, using glucose as carbon source. Microbes were injected and left incubating for six days and permeability was determined.

Coreflood experiments were also carried out to determine residual resistance factors $\left(\mathrm{F}_{\mathrm{rr}}\right)$. The aforementioned Berea cores were saturated with $0.5 \%$ brine solution and injected with 1 PV sucrose-peptone medium inoculated with NIPER 11. The cores were shut in for six days then flooded with brine, and flow rates and pressures were measured. The residual resistance factors $\left(\mathrm{F}_{\mathrm{rr}}\right)$ were then calculated using the following equation,

$$
\mathrm{F}_{\mathrm{rr}}=\left(\mathrm{Q}_{\mathrm{w}} / \Delta \mathrm{P}\right)_{\text {brine }} /\left(\mathrm{Q}_{\mathrm{w}} / \Delta \mathrm{P}\right)_{\text {AfterMicrobialInjection }} \quad \text { (21), here } \mathrm{Q}_{\mathrm{w}}
$$

is the flow rate, and $\Delta \mathrm{P}$ is the pressure difference between fluid input and output. The residual resistance factor is a measured of the stability of the biological plugging system following nutrient treatment and brine flush. It is also described as the mobility of the water-rich phase before treatment divided by the mobility of the water-rich after treatment,

$$
\mathrm{F}_{\mathrm{rr}}=(\mathrm{k} / \mu)_{\text {brine }} /(\mathrm{k} / \mu)_{\text {AfterMicrobialInjection }} \quad \text { (22), where } \mathrm{k}
$$

is the permeability and $\mathrm{m}$ is the viscosity of the flowing phase. Since the viscosity of the flowing phase does not change significantly before and after treatment, the expression simplifies to, 


$$
\mathrm{F}_{\mathrm{rr}}=(\mathrm{k})_{\text {brine }} /(\mathrm{k})_{\text {AfterMicrobiallnjection }}
$$

Figures 13 and 14 show the experimental results obtained for residual resistance factors for NIPER 11 and $1 \mathrm{~A}$ cultures. The best results were obtained using the backpressure regulator shown in Figure 1. NIPER 11 produced a significant increase in the residual resistance factors values for the entire range of flow values studied. The high residual resistance factor values are explained by a high microbial growth rate inside the core pores. These results were supported by permeability measurements before and after microbial injection. The residual resistance factor values decreased as the volumetric flow rate increased. This behavior is attributed to looser microbial attachment to the solid surface as flow rate increases. No significant differences in the experimental results were observed changing the nutrient sugar.

Figure 14 shows equivalent results for NIPER 1A cultures. Residual resistance factor values measured for NIPER 1A cultures were significantly lower than the ones measured for NIPER 11. It seems that NIPER 1A microbes only change the porosity of the cores due to cellular growth and not by high production of biopolymers as NIPER 11 cultures.

Experiments were also carried out using NIPER 1A cultures, and the modified medium E without yeast extract, $10 \mathrm{~g} / \mathrm{l}$ sucrose as carbon source, and $5 \mathrm{~g} / \mathrm{l}$ of $\mathrm{NaNO}_{3}$ as nitrate source. These cultures were found to produce a levan biopolymer when fermenting sucrose in absence of ammonium ion and yeast extract. Figure 15 shows typical results of these experiments. The residual resistance factor for NIPER 1A cultures feeding on the modified medium $\mathrm{E}$ plus sucrose and $\mathrm{NaNO}_{3}$ as nitrate source is almost three times as big as the values for the complete medium E plus yeast extract. The difference is attributed to increase permeability reduction produced by the formation of biopolymer by NIPER 1A under the modified culture medium. These results confirm the conclusions drawn in the chemical analysis section. NIPER 1A cultures can produce a biopolymer by nutrient manipulation. The product of this nutrient manipulation is an insoluble biopolymer that can reduce permeability in cores. 


\section{Task 4 -Process Simulation}

\section{Subtask 4.1 - UTCHEM Simulator}

A copy of UTCHEM, a chemical simulator developed by researchers at the University of Texas at Austin (Saad, $1989^{40}$, Delshad et al., 2002 ${ }^{41}$ ) was used by researchers at Prairie View A\&M University in this research. The simulator was used to model experiments and evaluate alternative treatments. Simulations were conducted to model oil mobilization by surfactant-producing microorganisms, and study permeability modification by microbial polymer systems.

The UTCHEM simulator does not have the ability to perform the required calculations in a single step; therefore, the required simulations should be obtained using a combination of steps. The microbial population was assumed to be already present in the simulation domain at a given base value. Nutrient injection was used to take the base value to the desired population value. The base value could be given as a concentration profile to simulate transport through the porous medium. The simulator accounts for cell growth, loose and attached, but does not take into account the porosity reduction by insoluble polymer production. This process was simulated by increasing cell size by a given factor. Biosurfactant production was simulated by using surfactant injection. The microbial injection, nutrient injection, growth, and bioproducts production should be simulated in several different steps using the fact that the simulator can be stopped, results recorded and a restart data file prepared. A typical procedure for injection of a surfactant producing bacteria followed by nutrient injection, incubation and waterflood is,

1. Simulated injection.

2. Nutrient injection.

3. Growth step.

4. Waterflood plus surfactant injection.

The waterflood should be accompanied of a surfactant injection step to simulate insitu biosurfactant production.

Several microbial/nutrient coreflood experiments were simulated. In all simulations, a 10-inch length, and 1 inch diameter core was used. NIPER 1A and NIPER 11 were the chosen microorganisms. A typical experiment for NIPER 1A was, injection of the core 
with 0.1 pore volume of microbes, and nutrient ( $4 \%$ molasses) followed by injection of 0.2 pore volumes of nutrient. The core was then shut-in for three days before the initiation of waterflood.

Parameters of the microbial system used in these simulations were determined from laboratory experiments or from simulation matches of laboratory tests using the developed microbial transport model by Chase et al. ${ }^{49}$, and Chang et al. ${ }^{50}$. A maximum growth rate of 8.4 day $^{-1}$ was determined for microorganism NIPER $1 \mathrm{~A}$ in the laboratory. The decay rate constant measured from the population change during the period of 600 hours is 0.22 day $^{-1}$. The cell yield coefficient, $\mathrm{Y}$, of 0.5 is computed from the nutrient consumption rate. The relative permeability values used were determined from a microbial coreflood in the laboratory ${ }^{47}$. Table 4 lists the core information, and microbial system parameters used in the simulation.

Figure16 shows the microbial population distribution along the Berea core after injection of 2 and $5 \mathrm{PV}$. The microbial population decreases along the core and waterflood due to the loss of microbes to rock adsorption. The distribution is more homogeneous as the water injection increases due to microbial transport through the core. Similar results are shown in Figure 17 for the nutrient concentration. The nutrient concentration decreased with length due to adsorption to the solid while it decreased with waterflood because of the microbial consumption. The profile becomes more homogeneous as the injection process progresses. Oil saturation profiles at different injection times are shown in Figure 18. The oil amount decreased constantly after the water injection started.

The reduction in permeability caused by microbial growth is shown in Figure 19. The permeability decreases following closely the microbial concentration profiles. It is higher where the microbial concentration is higher. It also becomes more homogeneous as the microbial distribution becomes more homogeneous. The permeability reduction values are also small reflecting the fact that NIPER $1 \mathrm{~A}$ is a poor polymer producer in this simulation. Figure 20 depicts the interfacial tension (IFT) profile along the core. There is also in this case a close relationship to the microbial concentration profile. The interfacial tension decreases as the microbial population increases. In some areas the IFT remains low despite the fact that the microbial population is also low due to transport of 
the biosurfactant through the core. Reed and Healy ${ }^{51}$ reported that an interfacial tension between oil and brine must reach $0.1 \mathrm{mN} / \mathrm{m}$ before any oil displacement begins and values of $0.01 \mathrm{mN} / \mathrm{m}$ or less are necessary for substantial oil production. Therefore, we can say based upon the criteria stated by Reed and Healy ${ }^{51}$ that there is surfactant production only in the front third of the core length. The surfactant is transported throughout the second third, and its concentration is very small in the last third.

Some simulations were run using data corresponding to NIPER 11 . NIPER 11 is a good polymer-producing microorganism; therefore, it produced higher permeability reduction than NIPER 1A. An example of this behavior is shown in Figure 21. The permeability is smaller in the front part of the core due to a non-homogeneous microbial population profile. The pore-blocking effect of the biopolymer was simulated by increasing the microorganisms size. The increased size was estimated using data taken from Jenneman et al. ${ }^{30}$. A comparison between experimental data and simulation results for oil recovery by a NIPER 11 culture is shown in Figure 22. The simulation results predict correctly the shape of the recovery curve. However, the predicted results are smaller than the measured data.

A proposed recovery strategy using NIPER 1A capacity of producing surfactant and polymer was simulated. This recovery strategy was simulated using the following steps:

1. Simulated injection.

2. Nutrient injection.

3. Growth step.

4. Waterflood plus surfactant injection.

5. Second nutrient injection.

6. Waterflood with polymer production.

Figure 23 shows the simulation results. Better recovery was obtained by switching to polymer production after the oil has been mobilized by decreasing capillary forces by biosurfactant production. This recovery strategy is equivalent to have a surfactant flood followed by a polymer sweep and it has been recognized as able to significantly improve oil recovery $^{52}$. Simulated results agreed well with experimental results. Better agreement was achieved simulating the biosurfactant production than the biopolymer production. 
The simulations results show that the UTCHEM simulator can be used as an effective tool to study microbial enhanced oil recovery. These results also show that metabolically controlled biochemical production can lead to better oil recovery.

\section{CONCLUSIONS}

Two microbial strains, NIPER $1 \mathrm{~A}$ and NIPER 11 were studied for their ability to produce biosurfactants and biopolymers when metabolically stimulated. Growth experiments showed that both microorganisms grow well on several culture media. NIPER 1A, surfactant producer, and NIPER 11, polymer producer, showed a lifetime cycle of more than 9 months when provided enough amount of nutrients. The lifecycle of both microorganisms reproduces the classical periods for bacterial growth.

NIPER 11 produces copious amount of biopolymer when fermenting sucrose; while it grows well, but it does not produce polymer when using glucose as carbon source. NIPER 1A produces good amount of biosurfactant when vitamins, enzymes and adequate nitrogen sources are available. Ammonium salts should be used as nitrogen source in this case plus yeast extract or trypticase/tryptone nutrients. NIPER 1A can produce some amount of biopolymer when grown from initial starvation conditions using sucrose as carbon source and sodium nitrate as nitrogen source without the vitamins, enzymes already mentioned. This finding allows alternate production of biosurfactant and biopolymer by metabolic control of NIPER 1A.

Commercial molasses can be used as carbon source with results similar to those using sucrose. Replacement of sucrose by molasses should result in significant economic savings.

Viscosity measurements showed that the biopolymer produced by NIPER 11 was practically insoluble in an aqueous phase. The biopolymer produced by NIPER 1A was also found to be insoluble in water solutions. NIPER 11 produces an insoluble polymer that causes significant permeability reduction, but it does not produce significant amounts of biosurfactant. NIPER 1A under the appropriate conditions produces a surfactant that can significantly reduce the interfacial tension between an aqueous phase, and an oil phase. Metabolic control of NIPER 1A produces a polymer that was found to reduce permeability in Berea cores. The measured permeability reduction is smaller than the 
values measured for NIPER 11, but higher than the data measured for surfactantproducing NIPER 1A cultures.

The residual recovery curves were similar for NIPER 1A and NIPER 11 samples using both light and heavy oils. Medium permeability cores produced more recovery than low permeability cores. High surfactant production is the main recovery mechanism during the NIPER 1A recovery experiments while improved sweep efficiency is the main recovery mechanism during the NIPER 11 experiments. These conclusions are supported by low surface tension values during the NIPER $1 \mathrm{~A}$ experiments and higher residual resistance factors during the NIPER 11 experiments.

NIPER 11 and NIPER 1A have low transport rates through the Berea cores. Specific strategies should be used to assure homogeneous distribution of microbial population. Growth of NIPER 11 using glucose as carbon source, and injection of NIPER 1A spores inside the porous medium; followed by nutrient stimulation seem to be the most appropriate placement strategies.

The UTCHEM commercial simulator can be used to simulate recovery processes including, microbial injection, nutrient injection, bio-products formation and waterfloods. The simulation should consist of several independent steps, modeled to achieve the desired effect.

Simulation results showed importance of microbial placement and microbial transport in porous media. The microbes tend to attach at the front of the core reducing permeability in that zone while the rest of the core does not show significant permeability reduction. Permeability reduction and surfactant production are mostly related to microbial population than to transport through the porous medium.

A recovery process combining several steps that resemble traditional surfactantpolymer flooding processes leads to improve recovery. This process includes, microbial injection, nutrient injection, microbial growth, surfactant production, nutrient activation and polymer production.

In conclusion: NIPER 1A cultures can produce a biopolymer by nutrient manipulation. The product of this nutrient manipulation is an insoluble biopolymer that can reduce permeability in cores. This property can be used to design better oil recovery processes. 


\section{REFERENCES}

1. Donaldson, E. C.; Chilingarian, G. V.; Yen, T. F. and -Sharma, M. K., “Chapter 1. Introduction” in Enhanced Oil Recovery, II, Processes and Operations, published by ELSEVIER - Amsterdam - Oxford - New York Tokyo, 1-12, 1989.

2. Bryant, R. S.; Donaldson, E. C.; G. V.; Yen and Chilingarian, G. V., "Chapter 14. Microbial Enhanced Oil Recovery," in Enhanced Oil Recovery, II, Processes and Operations, published by ELSEVIER - Amsterdam - Oxford New York - Tokyo, 423-450, 1989.

3. Beckman, J. W., Ind. Eng. Chem., 4(10),3, 1926.

4. ZoBell, C. E., "Bacteriological Process for Treatment of Fluid-Bearing Earth Formations,” U.S. Patent, No 2,413,278, Dec.24, 1946.

5. ZoBell, C. E., "Bacteria release of Oil from Sedimentary Materials,” Oil Gas J., 46(13), 62 (1947).

6. ZoBell, C. E., "Recovery of Hydrocarbons,” U.S. Patent, No 2,641,566, June 9, 1953.

7. Moses, V.; Robinson, J. P.; and Springham, D. G., "Microbial Enhancement of Oil Recovery in North Sea Reservoirs: A Requirement for Anaerobic Growth on Crude Oil," in Proceedings, International Conference on Microbial Enhancement of Oil Recovery, Oklahoma, May 16-21, 1983.

8. Bryant, R. S., Stepp, A. K.; Bertus, K. M.; Burchfield, T. E. and Dennis, M., “Microbial enhanced waterflooding field tests," Proc. of the 1994 SPE/DOE Improved Oil Recovery Symposium, April 17-20, 1994, Tulsa, OK, SPE/DOE Paper No. 27751.

9. Bryant, R. S., Stepp, A. K.; Bertus, K. M.; Burchfield, T. E. and Dennis, M., "Microbial enhanced waterflooding field pilots." DOE Report NIPEREPR/OP-93/8. 1993.

10. Lin, S. C., Sharma, M. M. and Georgiou, G., "Production and deactivation of biosurfactant by Bacillus licheniformis JF-2,” Biotechnol. Prog. 9:138-145, 1993.

11. Stepp, A. K.; Bryant, R. S.; Llave, F. M. and Lindsey, R. P., "Biopolymer system for permeability modification in porous media," Proc. of The Fifth 
International Conference on Microbial Enhanced Oil Recovery and Related Biotechnology for Solving Environmental Problems, September 11-14, 1995, Plano, TX.

12. Stepp, A. K., Bryant; R. S.; Llave, F. M.; Evans, D. B. and Bailey, S. A., "Microbial methods for improved conformance control in porous media," Proc. of the SPE/DOE Improved Oil Recovery Symposium, April 21-24, 1996, Tulsa, OK, SPE Paper 35357, 1996.

13. Cusack, F., Singh, S.; McCarthy; C.; Grieco, J.; De Rocco, M.; Nguyen, D.; Lappin-Scott, H. and Costerton, J. W., "Enhanced oil recovery - threedimensional sandpack simulation of ultramicrobacteria resuscitation in reservoir formation,” J. Gen. Microbiol., 138:647-655, 1992.

14. Bryant, R. S., and Burchfield, T. E., "Review of Microbial Technology for Improving Oil Recovery,” SPE Res. Eng. (May):151-154, 1989.

15. Singer, M. E., “Microbial Biosurfactants,” in Microbes and Oil Recovery, Bioresources Publications, El Paso, Texas, 19-38, 1985.

16. Brown, L. R., Vadie, A. A.; Stephens, J. O. and Azadpour, A., “Enhancement of the sweep efficiency of waterflooding operations by the in-situ microbial population of petroleum reservoirs," Proc. of The Fifth International Conference on Microbial Enhanced Oil Recovery and Related Biotechnology for Solving Environmental Problems, September 11-14, 1995, Plano, TX.

17. Jenneman, G. E., Gevertz, D.; Davey, M. E., Clark, J. B.; Wood, W. A.; Stevens, J. C. and Tankersley, C., "Development and application of microbial selective plugging processes,” Proc. of The Fifth International Conference on Microbial Enhanced Oil Recovery and Related Biotechnology for Solving Environmental Problems, September 11-14, 1995, Plano, TX.

18. Cooper, D.G., “Biosurfactants,” Microbiology Science, 3:145-149, 1986.

19. Syldatk, C. and Wagner, F., "Production of Biosurfactants. In: Biosurfactants and Biotechnolog,” Kosaric, N., Cairns, W.L., and Gary, N. C. C. (Eds). Marcel Decker, Inc., New York, 1987.

20. Riser-Roberts, E., Bioremediation of Petroleum Contaminated Sites, Boca Raton: C.K. Smoley. pp. 15-26, 1992.

21. Rosen, M.J., Surfactants and Interfacial Phenomena, $2^{\text {nd }}$ edition John Wiley \& Sons Inc. , New York., 1989. 
22. Guerra-Santos, L. H.; Kappeli, O.; and Fiecher, A. "Dependence of Pseudomonas-aeruginosa continuous culture biosurfactant production on nutritional and environmental factors,” Appl. Microbiol. Biotechnol., 123, 1986.

23. Arima, K.; Kakinuma, A.; and Tamura, G., "Surfactin, a crystalline peptidelipid surfactant produced by Bacillus subtilis: isolation, characterization and its inhibition of fibrin clot formation,” Biochem. Biophys. Res. Com. 31:488-494, 1968.

24. Zajic, J.E.; Guignard, H.; and Gerson, D.F., "Properties and biodegradation of a bioemulsifier from Corynebacterium hydrocarboclastus,” Biotechnol. Bioeng. , 19:1303-1320, 1977.

25. Finnerty, W.R.; and Singer, M.E., “A Microbial Biosurfactant - Physiology, Biochemistry, and Applications,” Dev. Ind. Microbiol., 1984.

26. Horowitz, S.; Gilbert, J.N.; and Griffin, W.M., "Isolation and characterization of a surfactant produced by Bacillus licheniformis,”, J. Ind. Microbiol., ㅁ:243248, 1990.

27. Beebe, J.L.; and Umbreit, W.W., "Extracellular lipid of Thiobacillus thioxidants,” J. Bacteriol., 82:788-789, 1971.

28. Javaheri, M.; Jenneman, G. E.; McInerney, M. J., and Knapp, R. M., "Anaerobic Production of a Biosurfactant by Bacillus licheniformis JF-2," Applied and Environ. Microbiology, 698-799, 1985.

29. McInerney, M.J.; Javaheri, M.; and Nagle, D.P., "Properties of the biosurfactant produced by Bacillus licheniformis strain JF-2.J," Ind. Microbiol. ㅁ:95-102, 1990.

30. Jenneman, G. E.; Lappan, R. E.; and Webb, R. H., "Bacterial Profile Modification with Bulk Dextran Gels Produced by In-Situ Growth and Metabolism of Leuconostoc Species,” SPE Journal, ㅁ⑷, 466-473, 2000.

31. Ramsay, J. A.; Cooper, D. G. and Neufeld, R. J., "Effects of oil reservoir conditions on the production of water-insoluble levan by Bacillus licheniformis,” Geomicrobiol. J. 7:155-165, 1989.

32. Bryant, R. S.; Burchfield, T. E.; Chase, K. L.; Bertus, K. M. and Stepp, A. K.,. "Optimization of microbial formulations for oil recovery: mechanisms of oil mobilization, transport of microbes and metabolites, and effects of additives," 
Proc of the 64th Annual Technical Conference and Exhibition of the Society of Petroleum Engineers, October 8-11, San Antonio, TX, SPE Paper 19686.

33. Lin, S. C.; Minton, M. A.; Sharma. M. M.; and Georgiou, G., "Structural and immunological characterization of a biosurfactant produced by Bacillus licheniformis JF-2,” Applied Environ. Microbiology, 60, 31-38, 1994.

34. Bae, J. H., Chambers, K. T.; and Lee, H. O., "Microbial profile modification using spores," Presented at the 69th Annual Technical Conference and Exhibition of the Society of Petroleum Engineers, September 25-28, New Orleans, LA, SPE Paper 28617, 1994.

35. Bryant, R. S. and Douglas, J., "Evaluation of Microbial Systems in Porous Media for Enhanced Oil Recovery,” SPE Reservoir Engineering, SPE Paper 16284, May 1988.

36. Fujimoto. Y. 'Kinetics of microbial growth and substrate consumption.” $J$. Theo. Biol., 5: 171-191, 1963.

37. Gaudy, A. F., Jr., E. T. Gaudy. "Microbiology for Environmental Scientists and Engineers,” McGraw Hill Book Co., New York, 1980.

38. National Research Council. "In Situ Bioremediation.” Water Science and Technology Board. Washington, D.C. National Academy Press, 1993.

39. Tsouris, C., “Interfacial Surface Tension Determination,” private communication, 2003.

40. Saad, N., "Field Scale Simulation of Chemical Flooding,” Ph. D. Dissertation, University of Texas, 1989.

41. Delshad, M.; Asakawa, K.; Pope, G. A.; and Sepehrnoori, K., “Simulations of Chemical and Microbial Enhanced Oil Recovery Methods," SPE paper No 75237, presented in the SPE/DOE Improved Oil Recovery Symposium held in Tulsa, Oklahoma, 13-17, April 2002.

42. Nelson, R. C. and Pope G. A. "Phase Relationships in Chemical Flooding," Society of Petroleum Engineering Journal, 18, 325-338(1978).

43. Hirasaki, G. J. “Application of the Theory of Multicomponent, Multiphase Displacement to Three-Component, Two-Phase Surfactant Flooding,” Society of Petroleum Engineering Journal, 21, 191-204(1981). 
44. Lappin-Scott, H.; Cusack, F.; and Costerton, J. W., "Nutrient resuscitation and growth of starved cells in sandstone cores: A novel approach to enhanced oil recovery ,” Applied Environ. Microbiology, 54, 1373-1382, 1988.

45. Jack, T. R. et al., "Microbial Plugging of Enhanced Oil Recovery,” Microbial Enhanced Oil Recovery, Developments in Petroleum Science, E. C. Donaldson, G. V. Chiligarian, and T. F. Yen (eds.), Elsevier Science Publishers, Amsterdam, 2, 125, 1993.

46. Lappan, R. E.; and Fogler, H. S., "Effect of Bacterial Polysaccharide Production on Formation Damage,” SPEPE, (May), 167, 1992.

47. Thompson, B. G.; and Jack, T. R., "Method of Enhancing Oil Recovery by Use of Exopolymer-Producing Microorganisms,” U.S. Patent No 4,561,500, 1985.

48. Parli, J. A.; Stepp, A. K.; Evans, D. B.; Bryant, R.S.; and Jenneman, G.E., "Transport and Stability of Polymer-Producing Bacteria in Porous Media," SPE paper No 39670, presented in the SPE/DOE Improved Oil Recovery Symposium, 19-22 April, Tulsa, Oklahoma, 1998.

49. Chase, K.L., R.S. Bryant, and T.E. Burchfield, K.M. Bertus, and A.K. Stepp, "Investigation of Microbial Mechanisms for Oil Mobilization in Porous Media.” Pres. at 1990 International Conference on Microbially Enhanced Oil Recovery, May 27 - June 1, 1990.

50. Chang, M-M., F. T. Chung, R.S. Bryant, H.W. Gao, and T.E. Burchfield, "Modeling and Laboratory Investigation of Microbial Transport Phenomena in Porous Media," SPE paper No 22845. Pres. at the $66^{\text {th }}$ SPE Annual Conference at Dallas, TX, Oct. 6-9, 1991.

51. Reed, R. L., and Healy, R. N., "Some Physicochemical Aspects of Microemulsion Flooding: A Review,” in D. O. Shah and R. S. Schechter, eds., Improved Oil Recovery by Surfactants and Polymer Flooding, Academic Press Inc., New York, 1977.

52. Alban, N., "Surfactant-Polymer Interactions in Oil Recovery," Master Thesis, Prairie View A\&M University, 2001. 
TABLES AND FIGURES

\begin{tabular}{|c|c|c|}
\hline Medium & Chemical & Amount $(\mathrm{g} / \mathbf{l})$ \\
\hline \multirow[t]{6}{*}{$\begin{array}{l}\text { Tryptic Soy Broth } \\
\text { (Difco 0370) }\end{array}$} & Tryptone or Trypticase & 17.0 \\
\hline & Soytone or Phytone & 3.0 \\
\hline & Sodium Chloride & 5.0 \\
\hline & $\begin{array}{l}\text { Dextrose } \\
\end{array}$ & 2.5 \\
\hline & Dipotassium Phosphate & 2.5 \\
\hline & $\begin{array}{c}\text { Reagent-Grade Distilled } \\
\text { Water }\end{array}$ & $1.0 \mathrm{~L}$ \\
\hline Preparation & $\begin{array}{c}30 \mathrm{~g} \text { of TSB to } 1 \mathrm{~L} \text { of } \\
\text { Distilled water }\end{array}$ & \\
\hline \multirow[t]{7}{*}{$\begin{array}{c}\text { Thioglycolate Broth } \\
\text { (Ref. 2-186) }\end{array}$} & Peptone from Casein & 15.0 \\
\hline & Yeast Extract & 5.0 \\
\hline & Sodium Chloride & 2.5 \\
\hline & Dextrose & 5.5 \\
\hline & Sodium Thioglycolate & 0.5 \\
\hline & L-Cystine & 0.5 \\
\hline & $\begin{array}{c}\text { Reagent-Grade Distilled } \\
\text { Water }\end{array}$ & $1.0 \mathrm{~L}$ \\
\hline Preparation & $\begin{array}{l}29 \mathrm{~g} \text { of ThB to } 1 \mathrm{~L} \text { of } \\
\text { Distilled water }\end{array}$ & \\
\hline Medium E & Sucrose & 10.0 \\
\hline \multirow[t]{7}{*}{ (ATCC 1502) } & Ammonium Sulfate & 1.0 \\
\hline & Sodium Chloride & 50.0 \\
\hline & Magnesium Sulfate & 0.25 \\
\hline & Dipotassium Phosphate & 10.6 \\
\hline & Potassium Phosphate & 5.3 \\
\hline & Trace Salts & $10 \mathrm{ml}$ \\
\hline & Distilled Water & $1.0 \mathrm{~L}$ \\
\hline Preparation & $76.2 \mathrm{~g}$ to $1 \mathrm{~L}$ of Distilled & \\
\hline \multirow[t]{5}{*}{ Modified Medium E } & Sodium Chloride & 50.0 \\
\hline & Magnesium Sulfate & 0.25 \\
\hline & Dipotassium Phosphate & 10.6 \\
\hline & Potassium Phosphate & 5.3 \\
\hline & Distilled Water & $1.0 \mathrm{~L}$ \\
\hline
\end{tabular}

Table 1. Summary of culture media. 


\begin{tabular}{|l|c|c|c|c|c|}
\hline Microbe & Time & Nutrient & $\begin{array}{c}\text { SPC/ml } \\
\text { (c.f.u.) }\end{array}$ & $\begin{array}{c}\text { Viscosity } \\
\text { (cp) }\end{array}$ & $\begin{array}{c}\text { Surface } \\
\text { Tension } \\
\text { (mN/m) }\end{array}$ \\
\hline NIPER 1A & 1 week & Molasses & $1.10 \mathrm{E}+07$ & 1.05 & 29 \\
\hline NIPER 1A & 1 week & Sucrose & $1.20 \mathrm{E}+07$ & 1.08 & 28 \\
\hline NIPER 1A & 1 week & Glucose & $3.50 \mathrm{E}+06$ & 1.05 & 51 \\
\hline Blank & 1 week & NA & NA & 1.02 & 68 \\
\hline & & & & & \\
\hline NIPER 11 & 1 week & Molasses & $1.15 \mathrm{E}+07$ & 1.187 & 62 \\
\hline NIPER 11 & 1 week & Sucrose & $1.20 \mathrm{E}+07$ & 1.176 & 64 \\
\hline Blank & 1 week & NA & NA & 1.020 & 68 \\
\hline
\end{tabular}

Table 2. Summary of typical laboratory experiments.

\begin{tabular}{|c|c|c|c|c|c|c|c|c|c|}
\hline Oil & Microbe & Sugar & $\mathrm{k}$ & $\mathrm{SPC} / \mathrm{ml}$ & $\begin{array}{c}\text { Viscosity } \\
(\mathrm{md})\end{array}$ & $\begin{array}{c}\text { Surface } \\
\text { Tension } \\
(\mathrm{mN} / \mathrm{m})\end{array}$ & $\begin{array}{c}\mathrm{S}_{\text {orwf }} \\
(\% \mathrm{PV})\end{array}$ & $\begin{array}{c}\mathrm{S}_{\text {orf }} \\
(\% \mathrm{PV})\end{array}$ & $\begin{array}{c}\mathrm{E}_{\mathrm{r}} \\
(\%)\end{array}$ \\
\hline $\begin{array}{c}\text { Delaware- } \\
\text { Childers }\end{array}$ & $\begin{array}{c}\text { NIPER } \\
1 \mathrm{~A}\end{array}$ & Sucrose & 180 & $2.1 \mathrm{E}+6$ & 1.0 & 29 & 25 & 11.7 & 53.2 \\
\hline $\begin{array}{c}\text { Delaware- } \\
\text { Childers }\end{array}$ & $\begin{array}{c}\text { NIPER } \\
1 \mathrm{~A}\end{array}$ & Molasses & 165 & $1.9 \mathrm{E}+6$ & 1.0 & 31 & 24.5 & 11.3 & 53.9 \\
\hline $\begin{array}{c}\text { Delaware- } \\
\text { Childers }\end{array}$ & $\begin{array}{c}\text { NIPER } \\
1 \mathrm{~A}\end{array}$ & Glucose & 152 & $1.1 \mathrm{E}+6$ & 1.05 & 52 & 25.4 & 18.7 & 57.9 \\
\hline $\begin{array}{c}\text { Delaware- } \\
\text { Childers }\end{array}$ & $\begin{array}{c}\text { NIPER } \\
11\end{array}$ & Sucrose & 446 & $1.6 \mathrm{E}+6$ & 1.2 & 66 & 23.6 & 14.9 & 36.9 \\
\hline $\begin{array}{c}\text { Delaware- } \\
\text { Childers }\end{array}$ & $\begin{array}{c}\text { NIPER } \\
11\end{array}$ & Molasses & 465 & $1.5 \mathrm{E}+6$ & 1.25 & 64 & 24.8 & 15.8 & 36.3 \\
\hline $\begin{array}{c}\text { Delaware- } \\
\text { Childers }\end{array}$ & $\begin{array}{c}\text { NIPER } \\
11\end{array}$ & Glucose & 411 & $1.45 \mathrm{E}+6$ & 1.2 & 63 & 26.1 & 17.4 & 37.2 \\
\hline Chaffee & $\begin{array}{c}\text { NIPER } \\
1 \mathrm{~A}\end{array}$ & Sucrose & 432 & $1.5 \mathrm{E}+6$ & 1.03 & 29 & 39.7 & 11.1 & 72.0 \\
\hline Chaffee & $\begin{array}{c}\text { NIPER } \\
11\end{array}$ & Sucrose & 716 & $1.4 \mathrm{E}+6$ & 1.3 & 64 & 42.0 & 22.2 & 47.1 \\
\hline \hline
\end{tabular}

Table 3. Summary of typical recovery coreflood experiments. 


\begin{tabular}{|c|c|}
\hline Parameters & Values \\
\hline Core Volume & $128.6 \mathrm{~cm}^{3}$ \\
\hline Pore Volume & $27.8 \mathrm{~cm}^{3}$ \\
\hline Porosity & 0.216 \\
\hline Permeability & $526 \mathrm{mD}$ \\
\hline Injection rate & 1.5 (ft/day) \\
\hline Diffusion coefficient, microbes & $0.0055\left(\mathrm{ft}^{2} /\right.$ day $)$ \\
\hline Diffusion coefficient, nutrient & $0.0083\left(\mathrm{ft}^{2} /\right.$ day $)$ \\
\hline Declogging rate constant & $37\left(\right.$ day $\left.^{-1}\right)$ \\
\hline Cell yield coefficient (Y) & 0.5 \\
\hline Monod half growth constant & 0.5 or $6.23\left(\mathrm{lb} / \mathrm{ft}^{3}\right)$ \\
\hline Maximum growth rate & $8.4\left(\right.$ day $\left.^{-1}\right)$ \\
\hline Decay rate & $0.22\left(\right.$ day $\left.^{-1}\right)$ \\
\hline Chemotaxis Coefficient & 0 \\
\hline
\end{tabular}

Table 4. Microbial system parameters used in NIPER 1A simulations

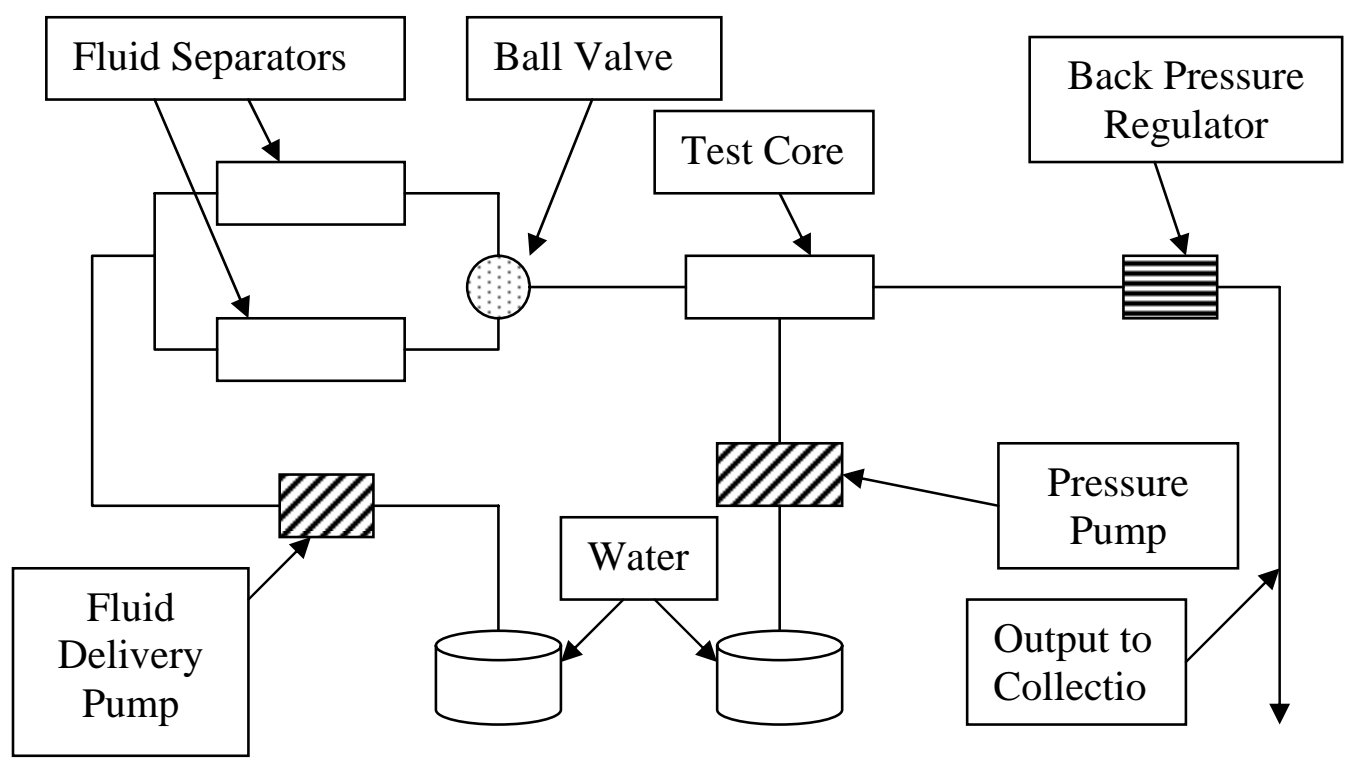

Figure 1. Experimental set-up for oil recovery experiments. 


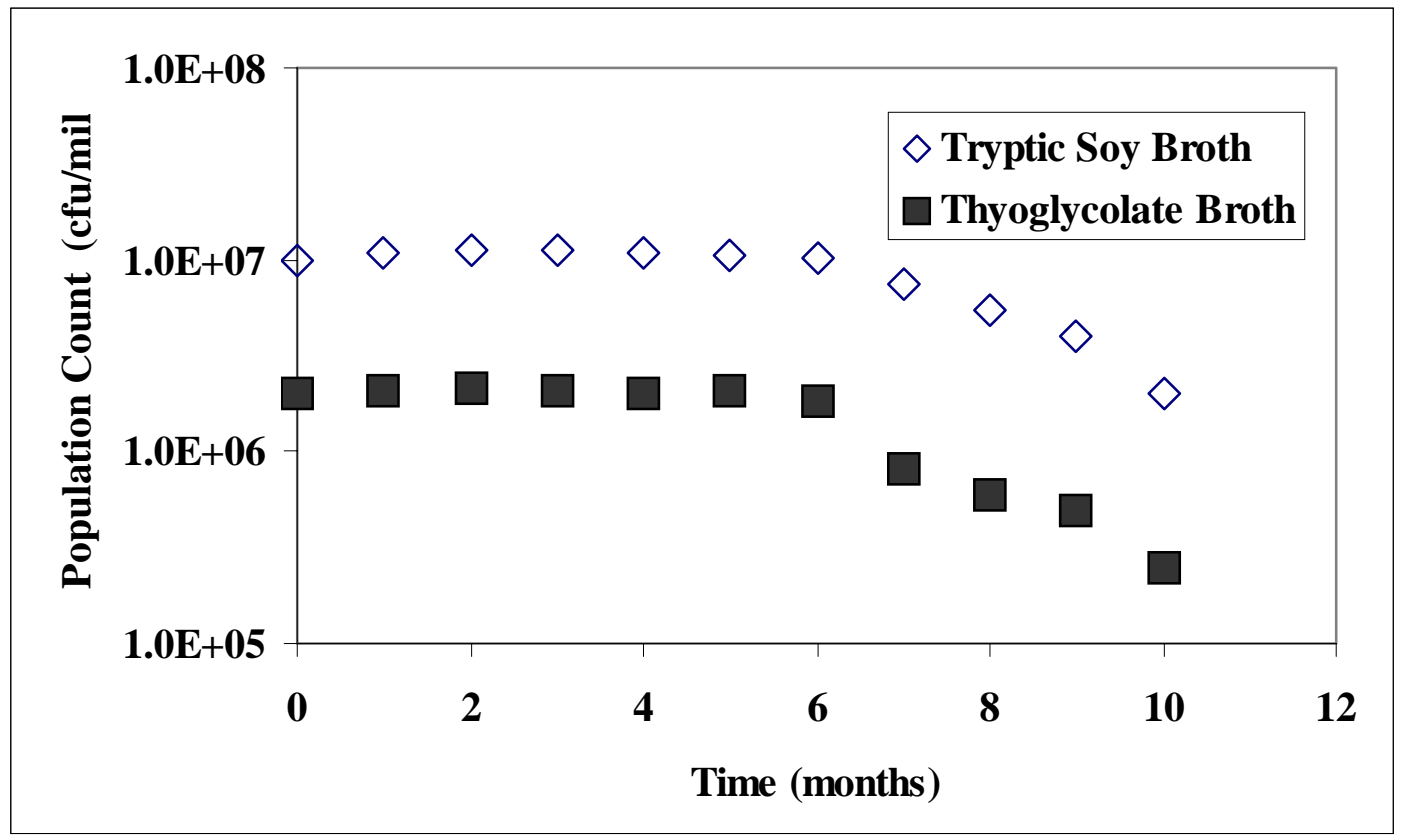

Figure 2. Lifetime cycle for NIPER 1A microorganisms in different culture media.

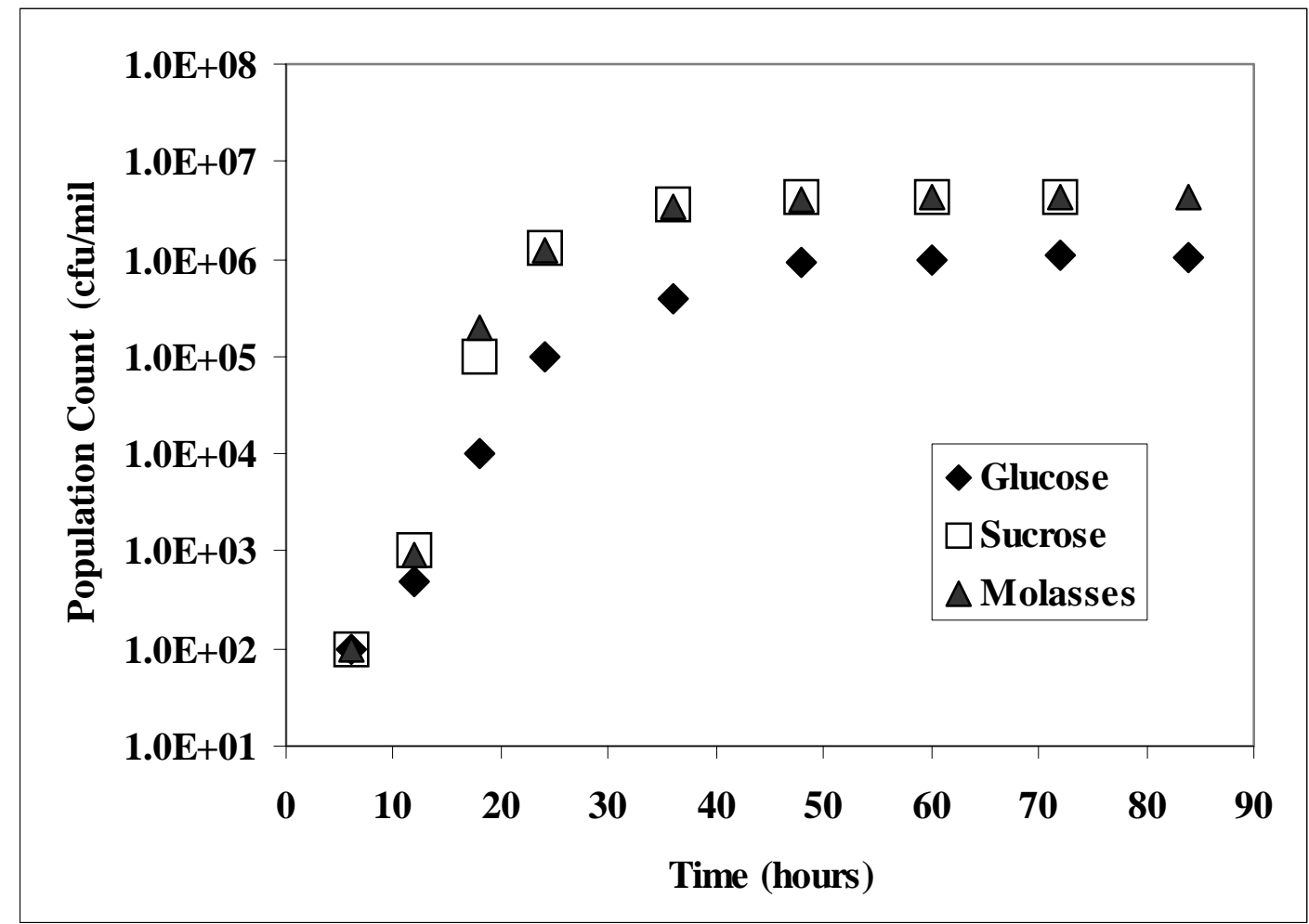

Figure 3. Growth curve for NIPER 1A microorgamisms, and different carbon sources. 


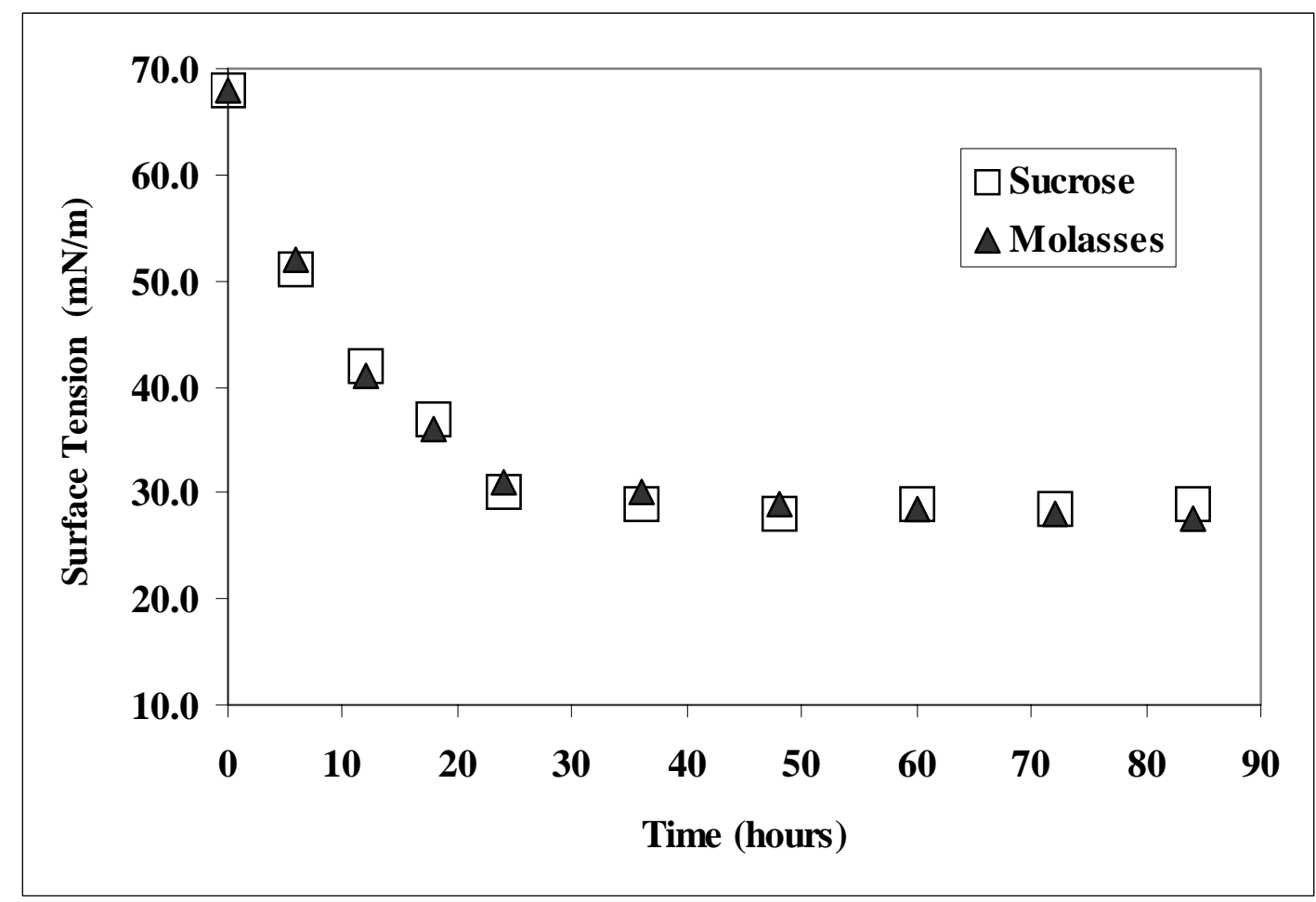

Figure 4. Surface tension reduction as a function of growth time for different carbon sources.

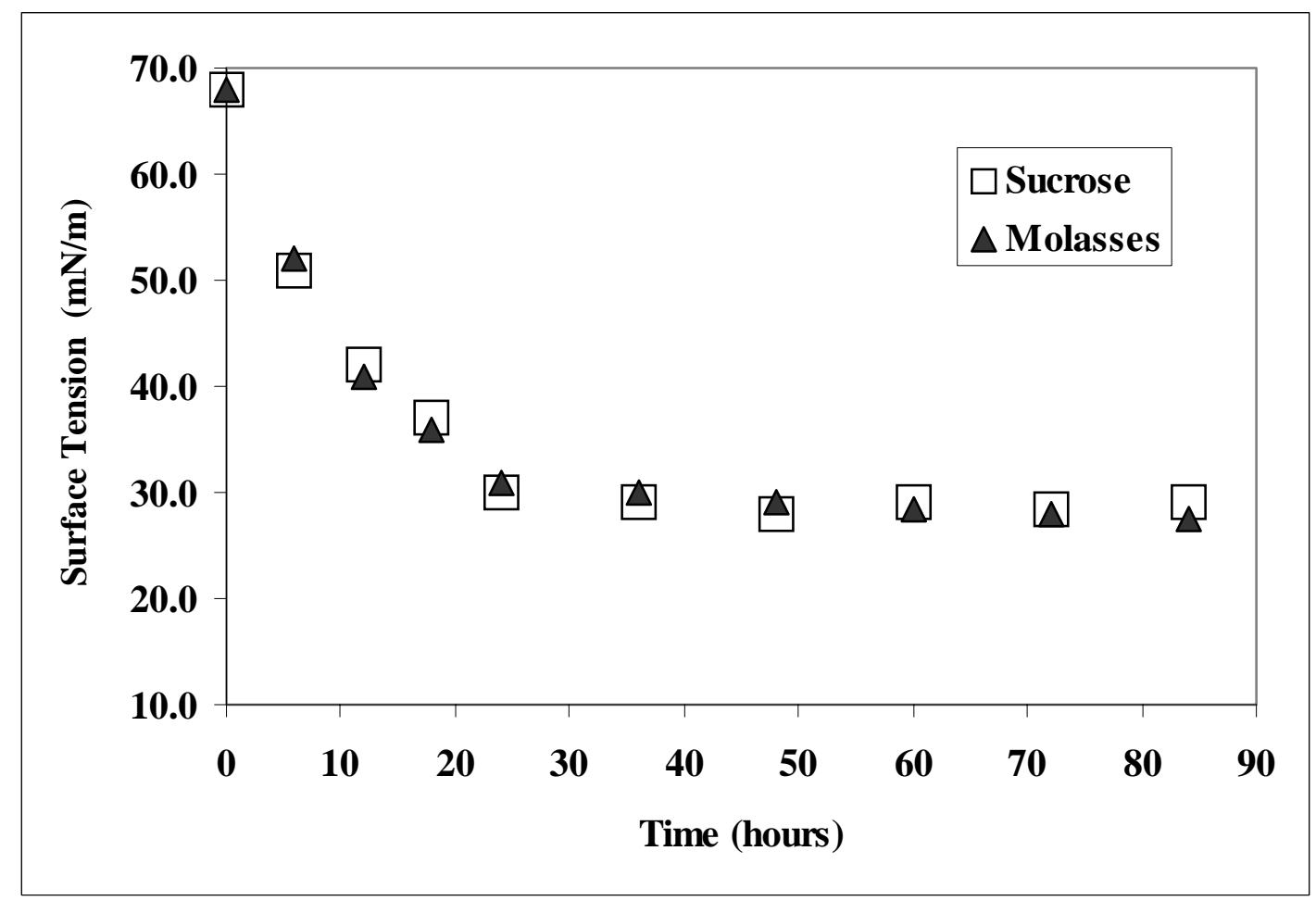

Figure 5. Interfacial tension reduction as a function of growth time for different carbon sources. 


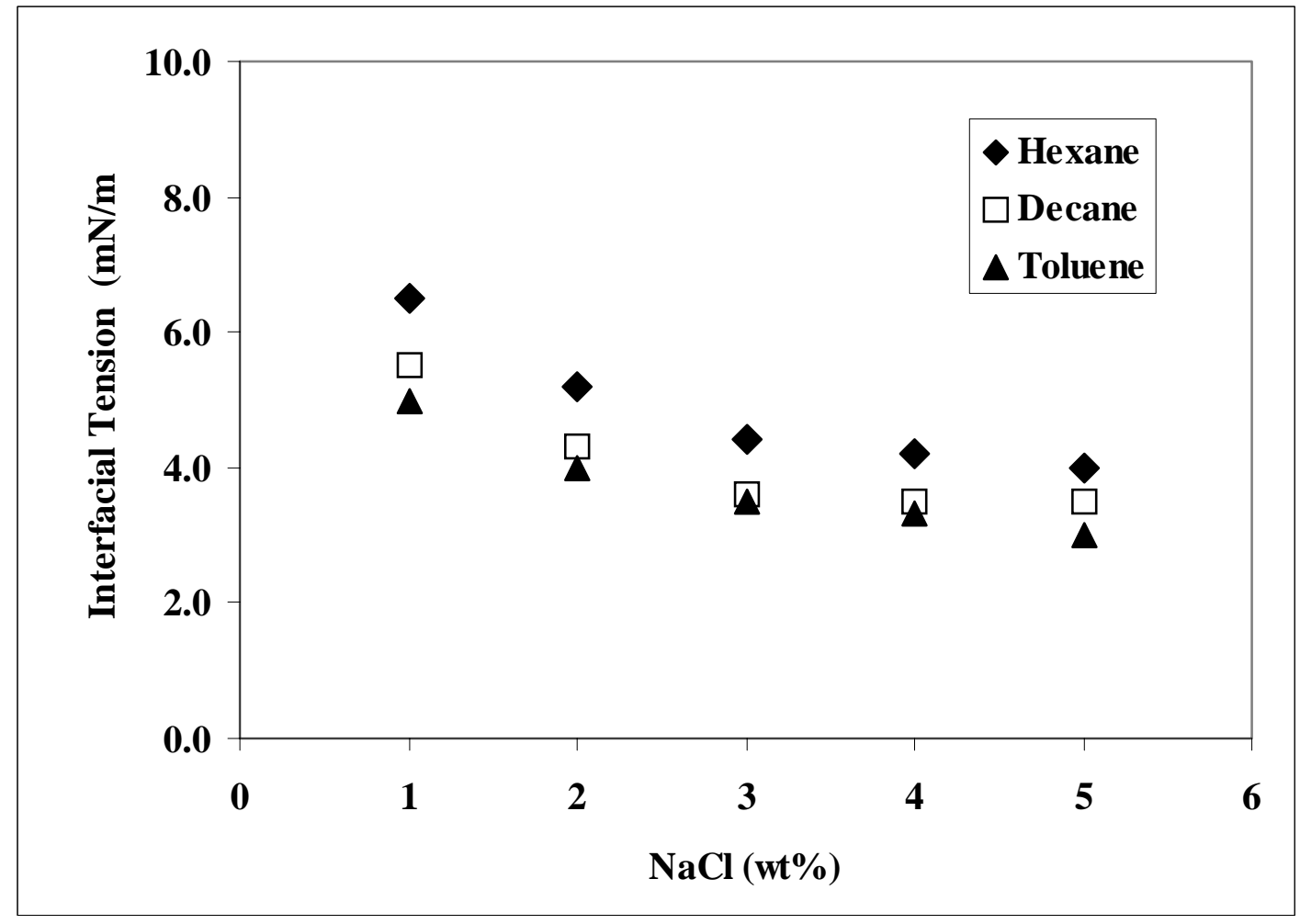

Figure 6. Interfacial tension reduction as a function of brine concentration for different oil phases.

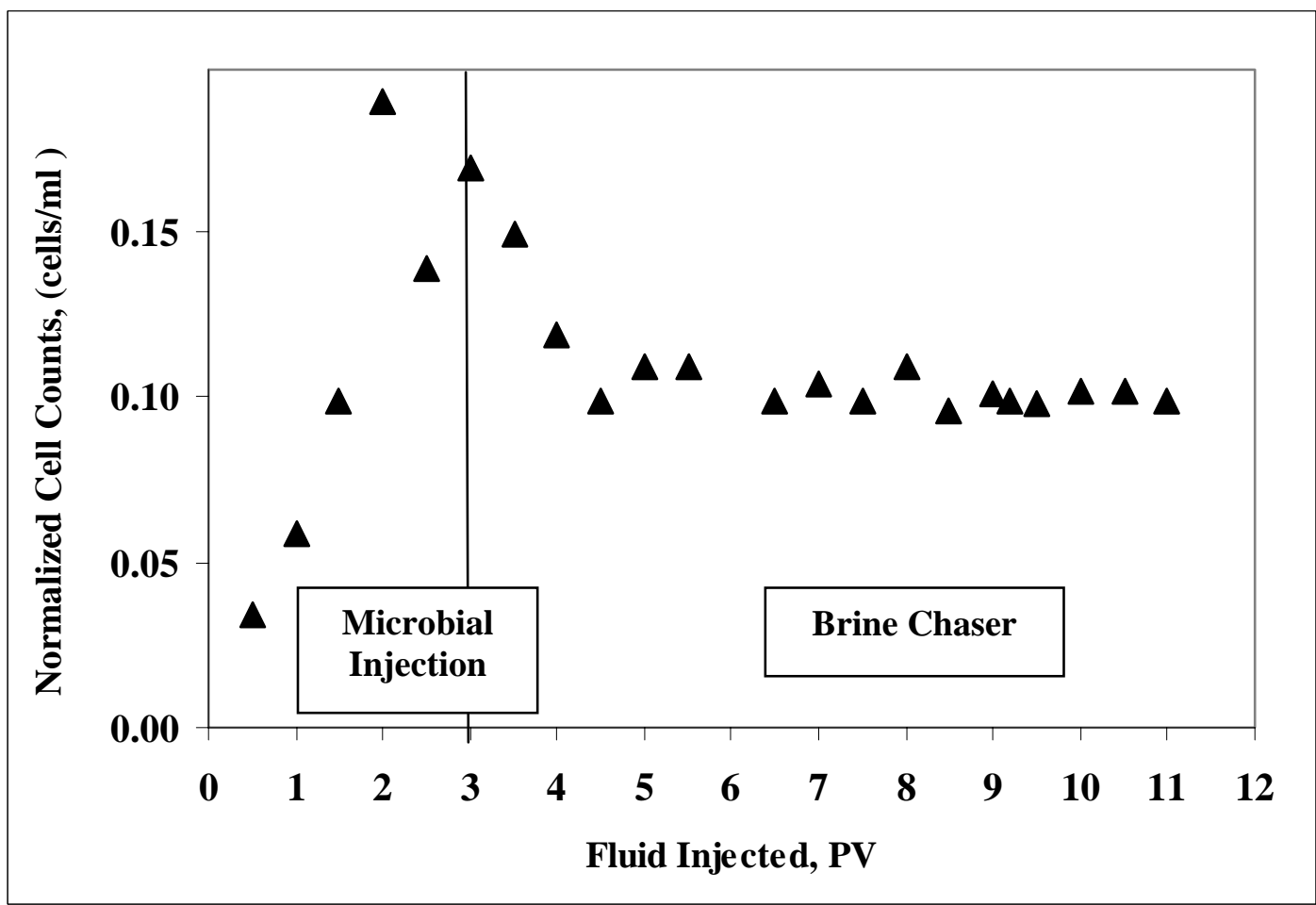

Figure 7. NIPER 11 retention in Berea cores, 3 PV microbial injection. 


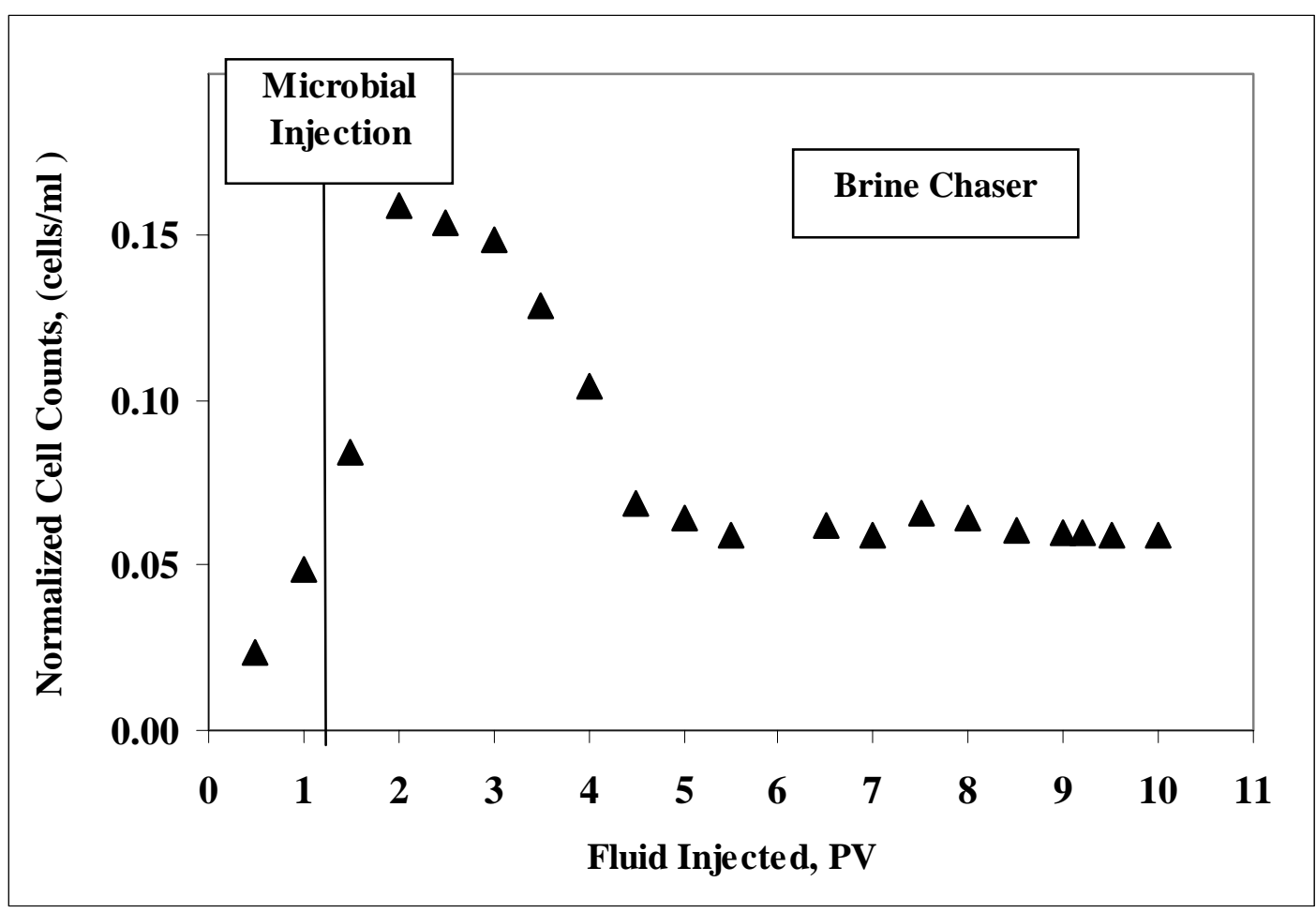

Figure 8. NIPER 1A retention in Berea cores, 1.2 PV microbial injection.

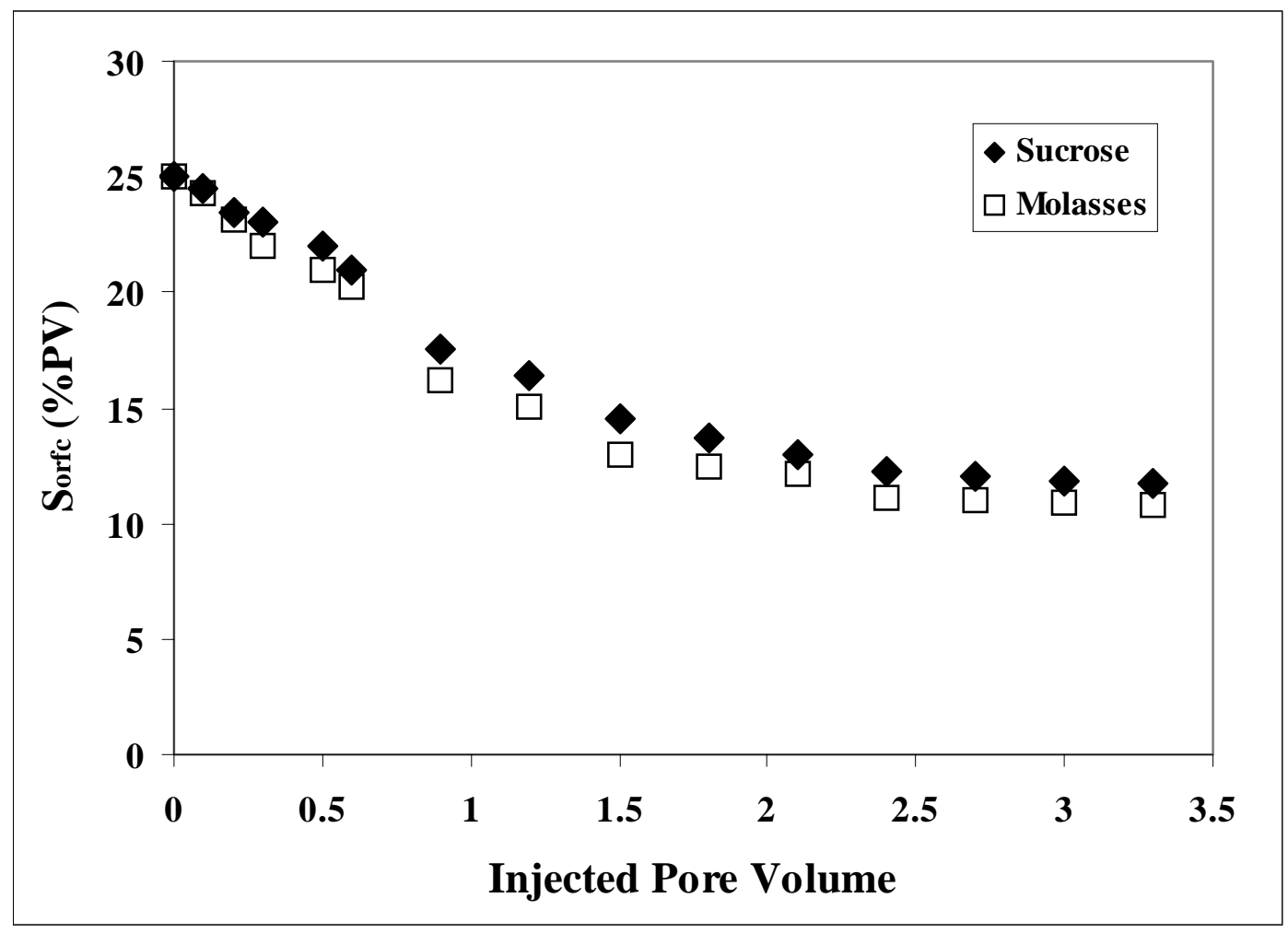

Figure 9. Residual oil saturation versus injected PV for NIPER 1A cultures. 


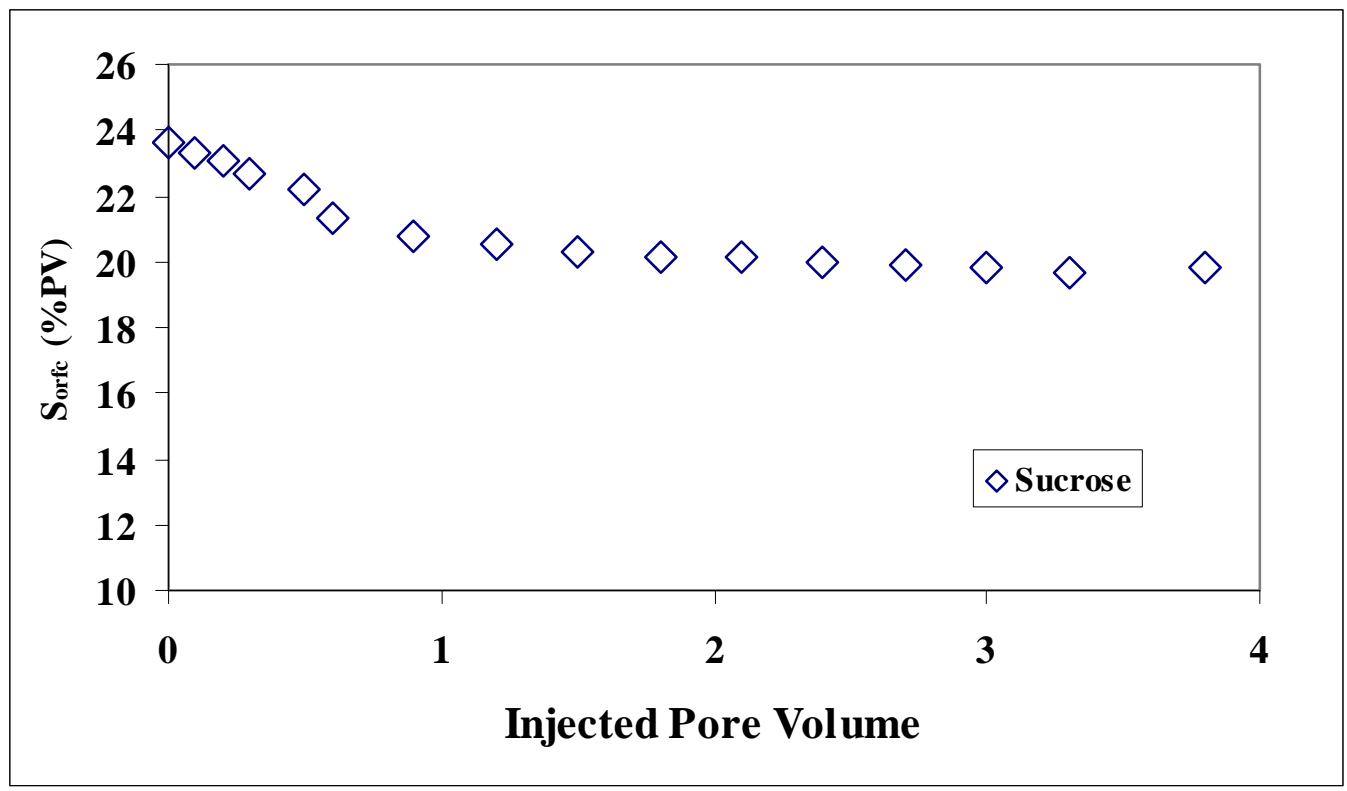

Figure 10. Residual oil saturation versus injected PV for NIPER 11 cultures.

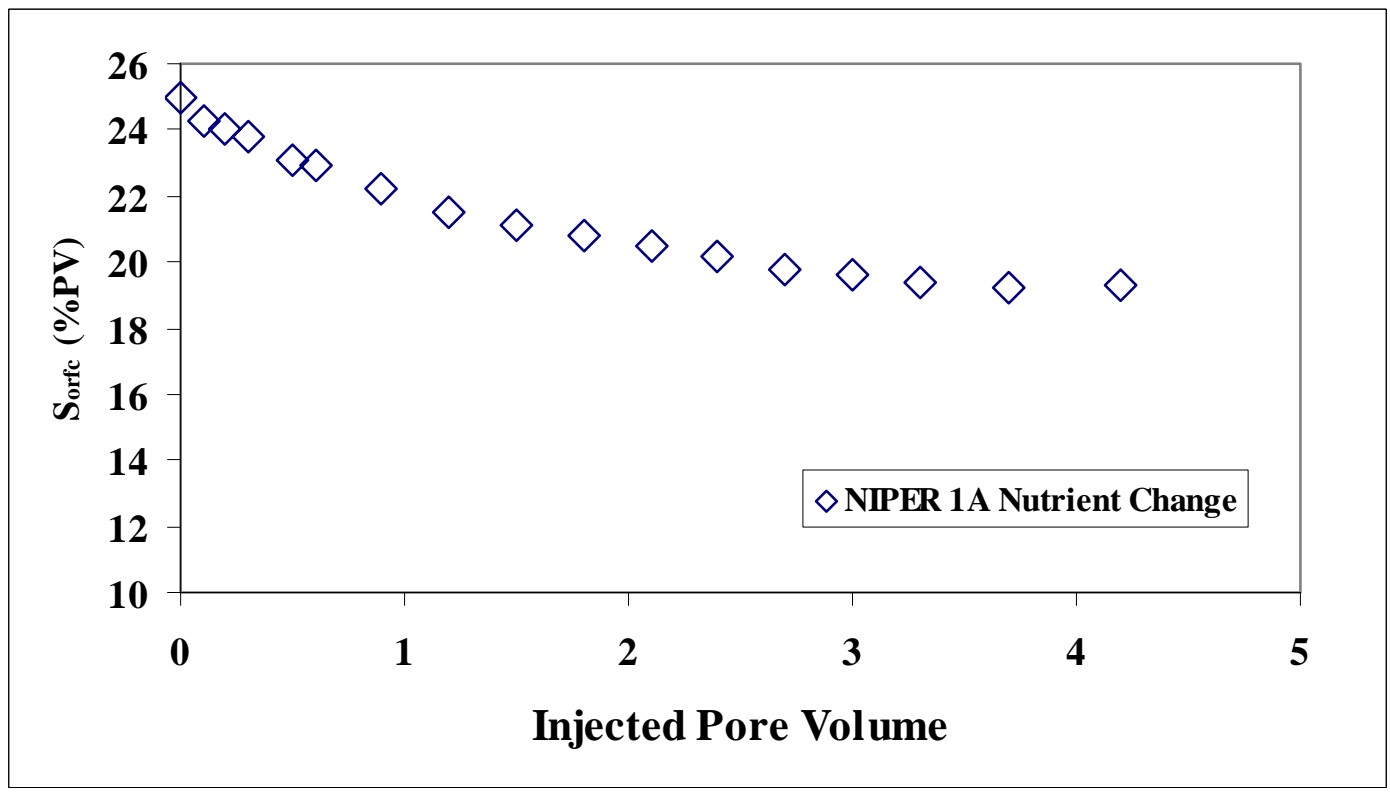

Figure 11. Residual oil saturation versus injected PV for NIPER 1A cultures using modified medium E plus sodium nitrate. 


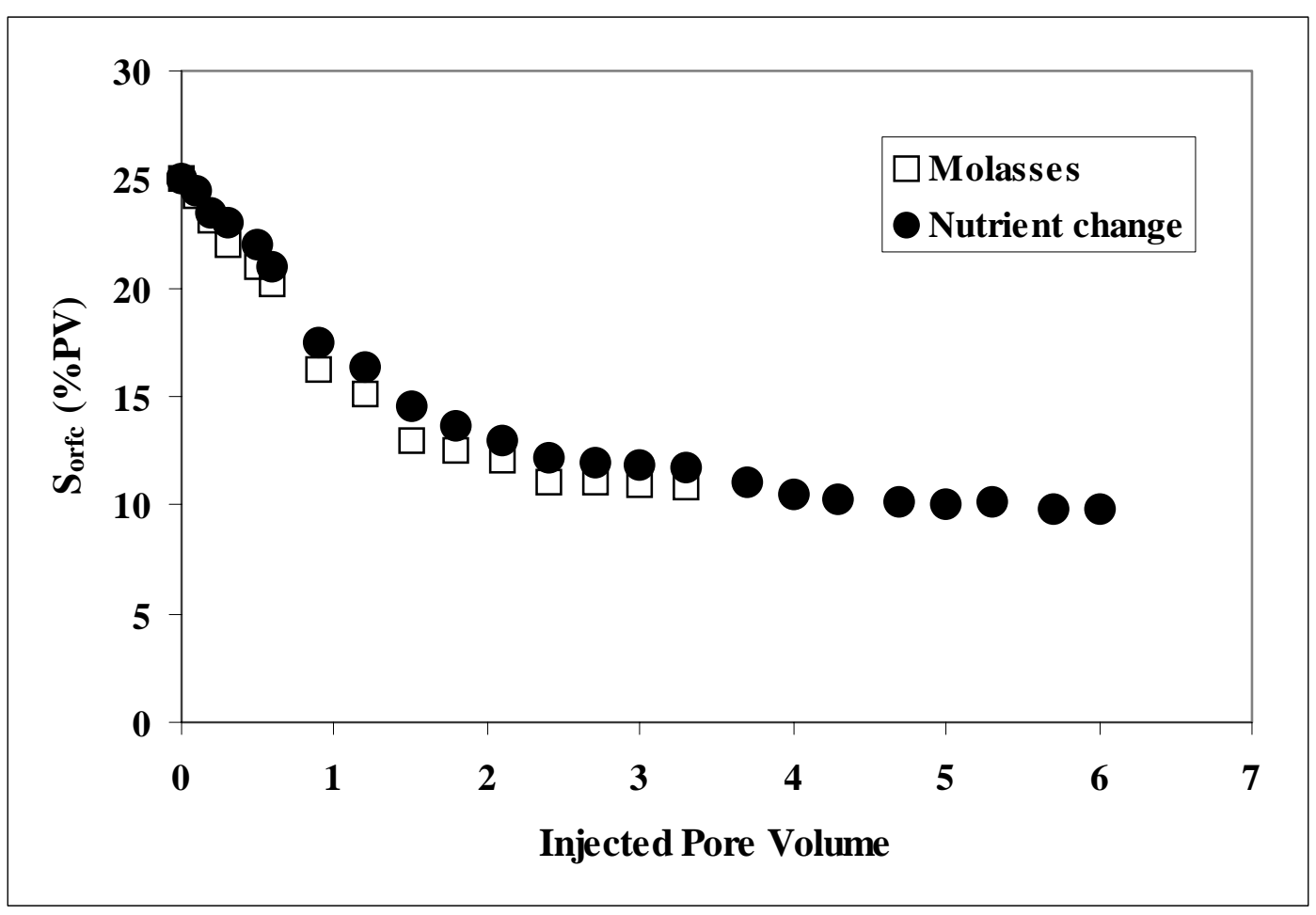

Figure 12. Residual oil saturation versus injected PV for NIPER 1A cultures using a combination of nutrients.

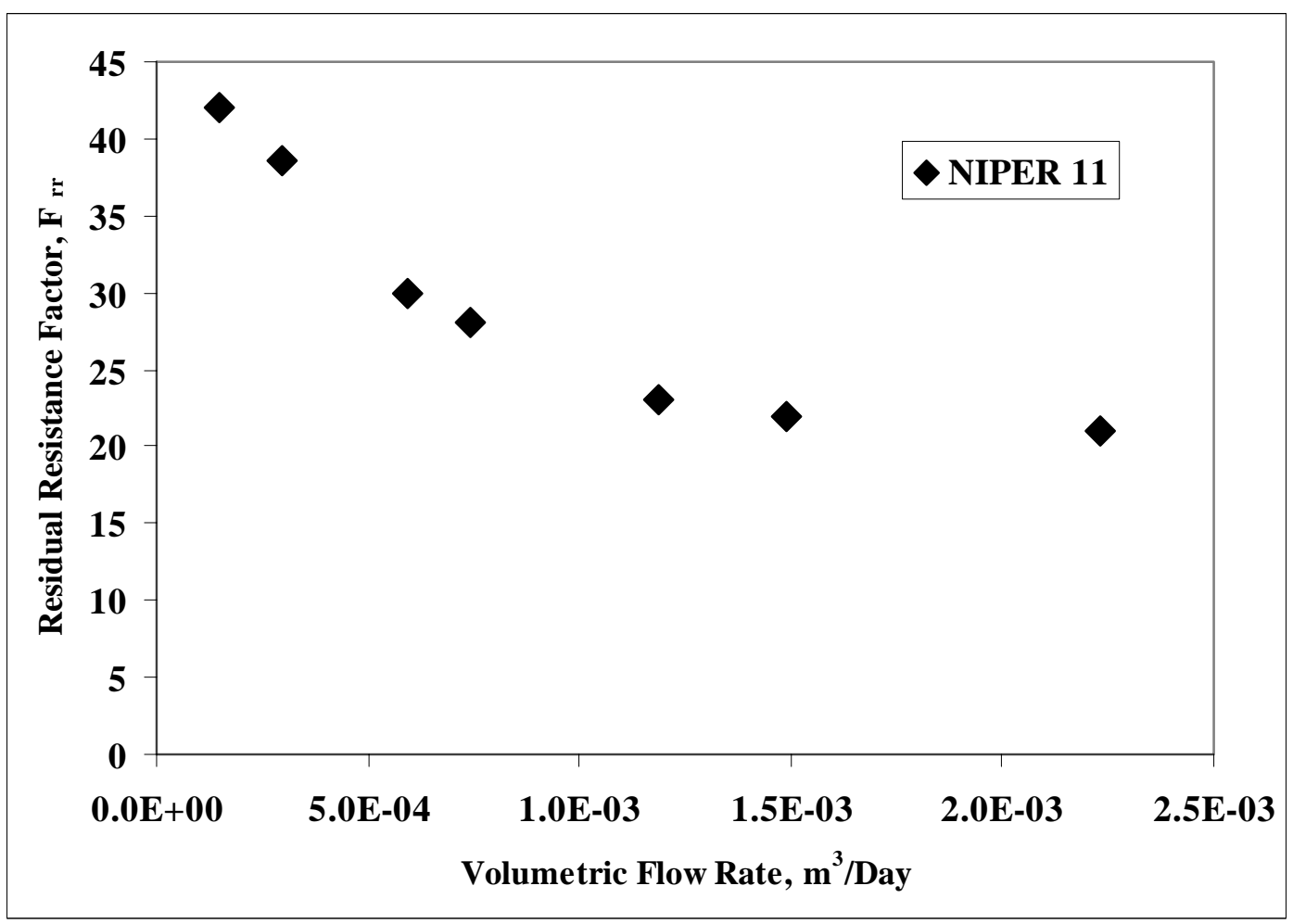

Figure 13. Residual resistance factor as a function of volumetric flow for NIPER 11 cultures. 


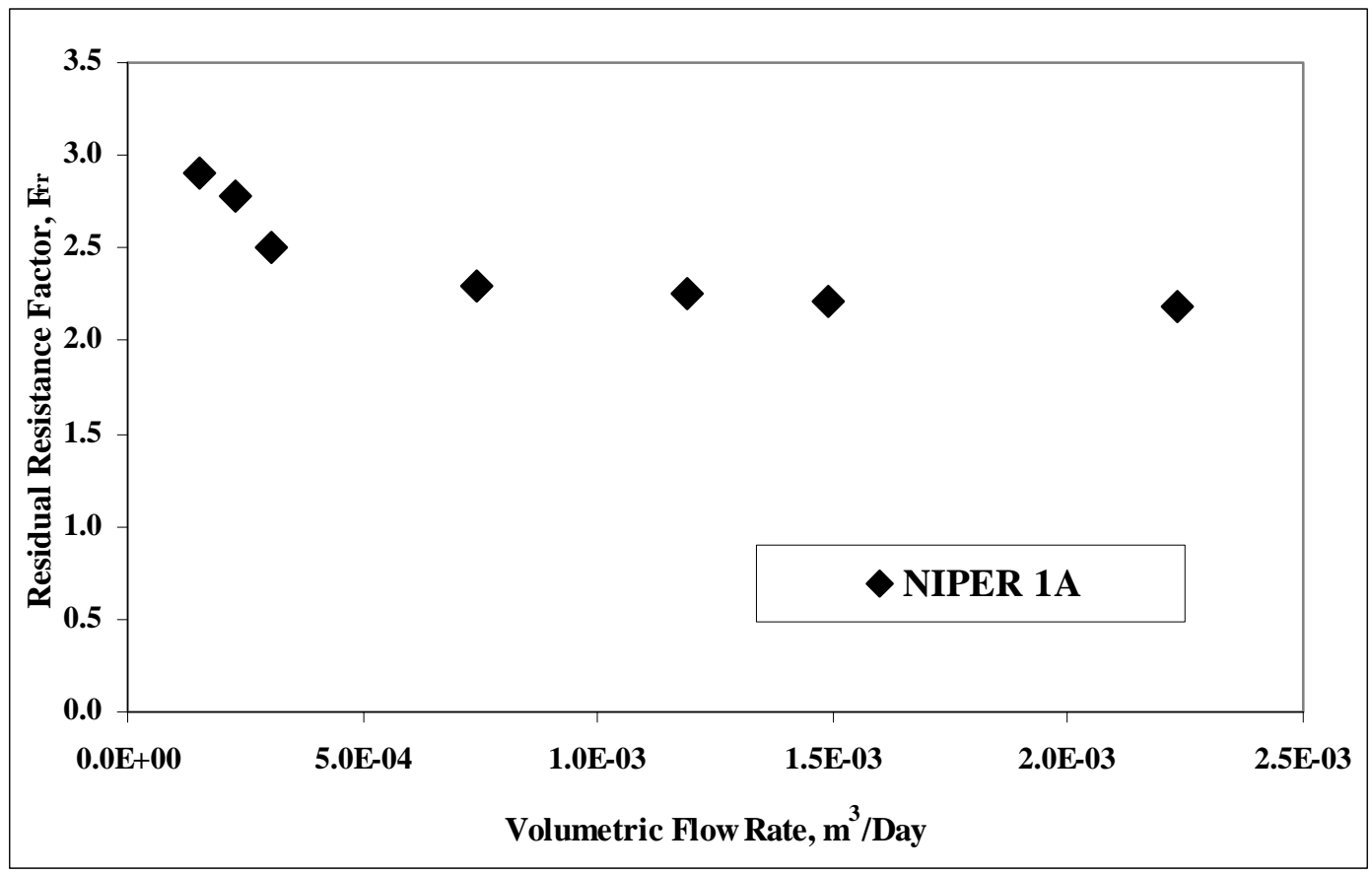

Figure 14. Residual resistance factor as a function of volumetric flow for NIPER 1A cultures using medium E plus yeas extract.

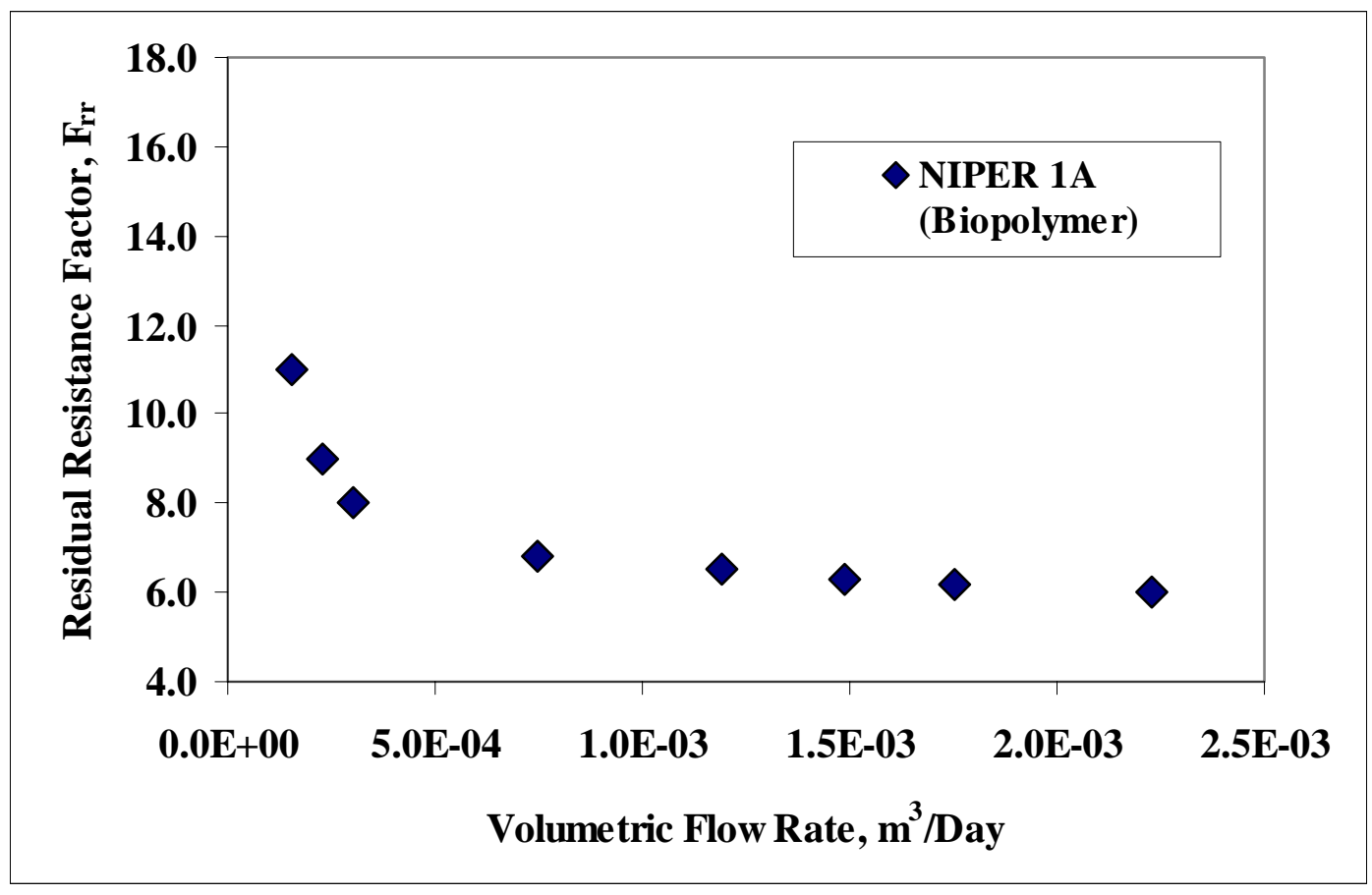

Figure 15. Residual oil saturation versus injected flow rate for NIPER 1A cultures using sodium nitrate. 


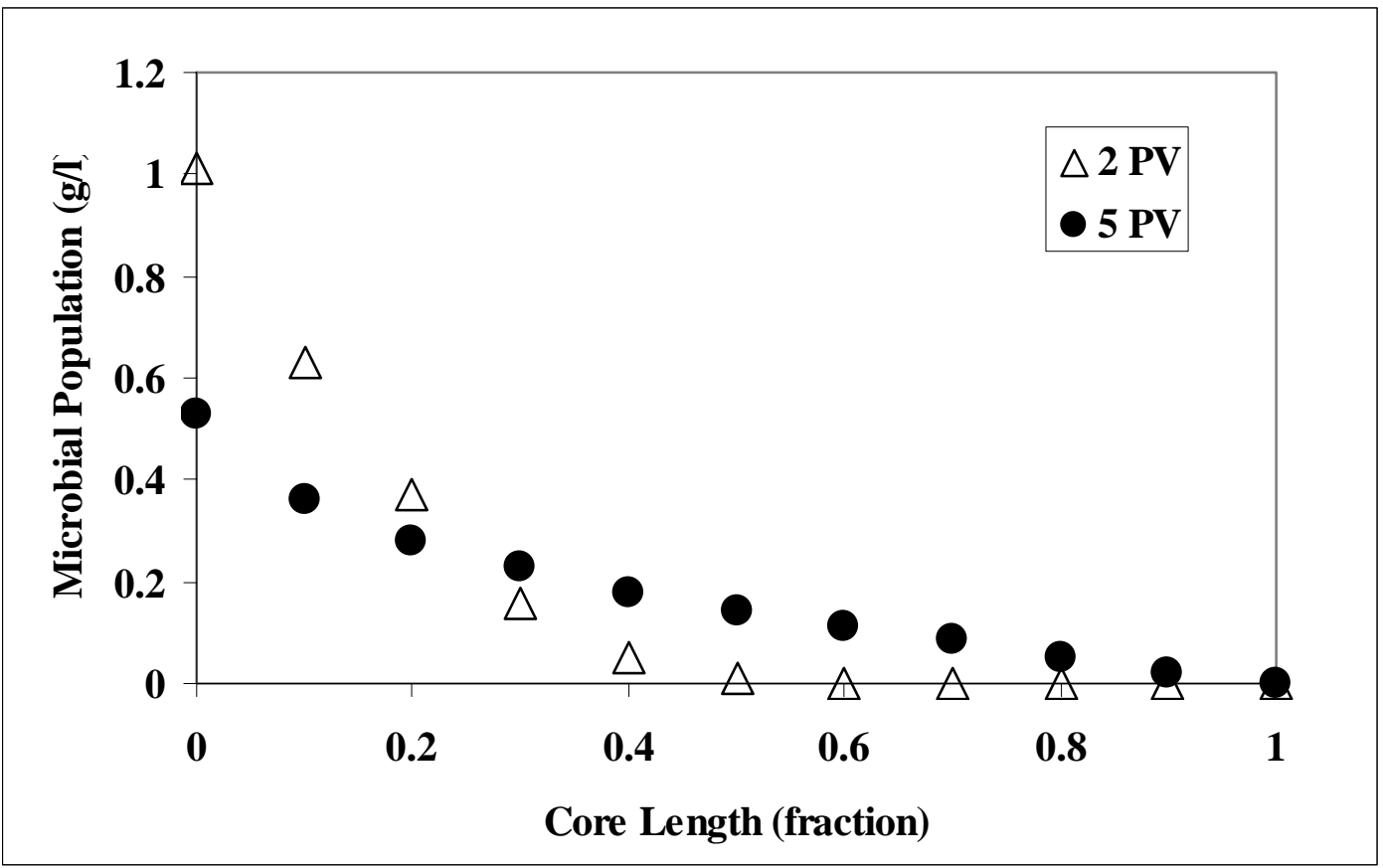

Figure 16. NIPER 1A microbial distribution in Berea cores after 2 and 5 PV injection.

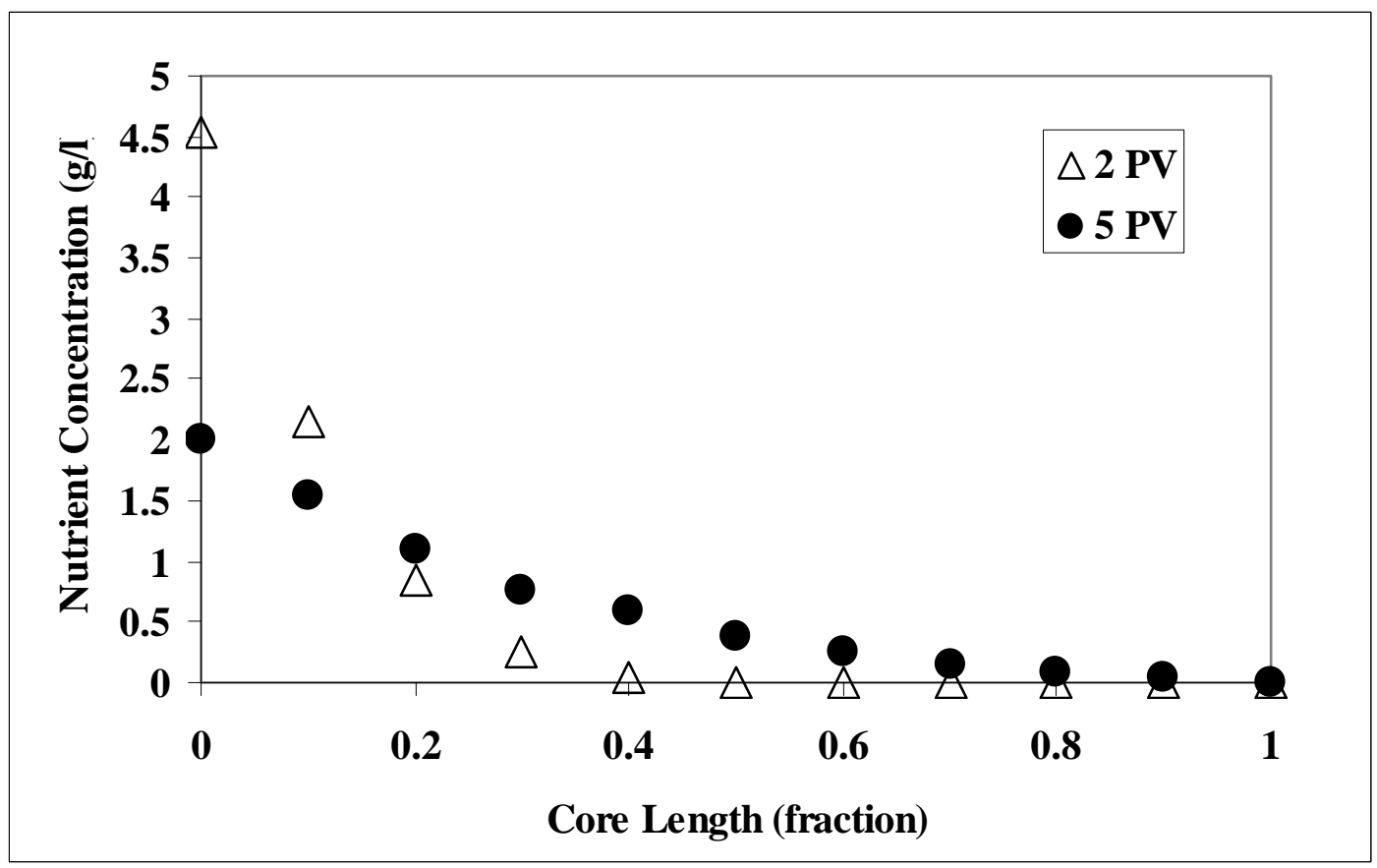

Figure 17. Nutrient distribution in Berea cores after 2 and 5 PV injection. 


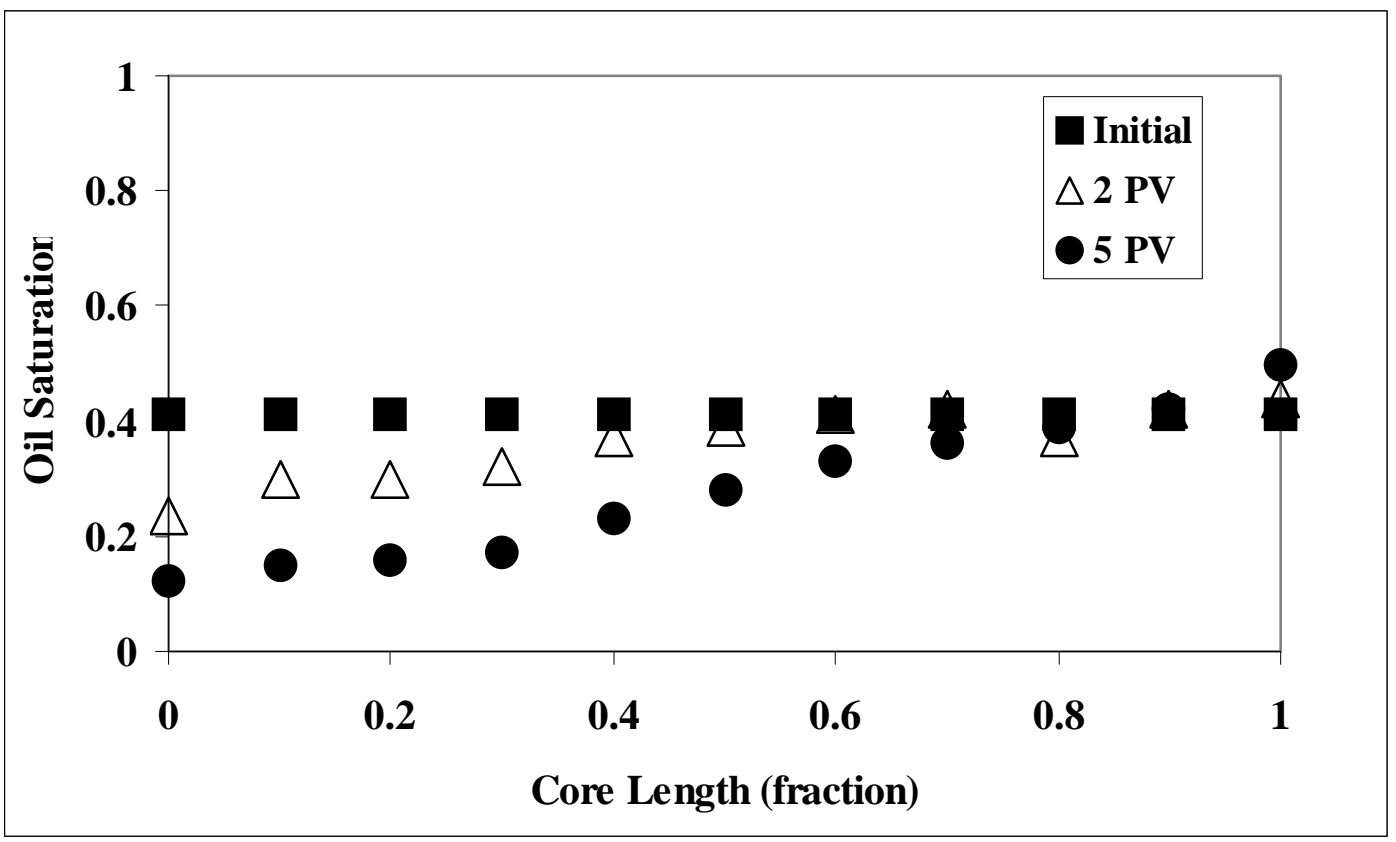

Figure 18. Oil saturation profiles for different injection volumes.

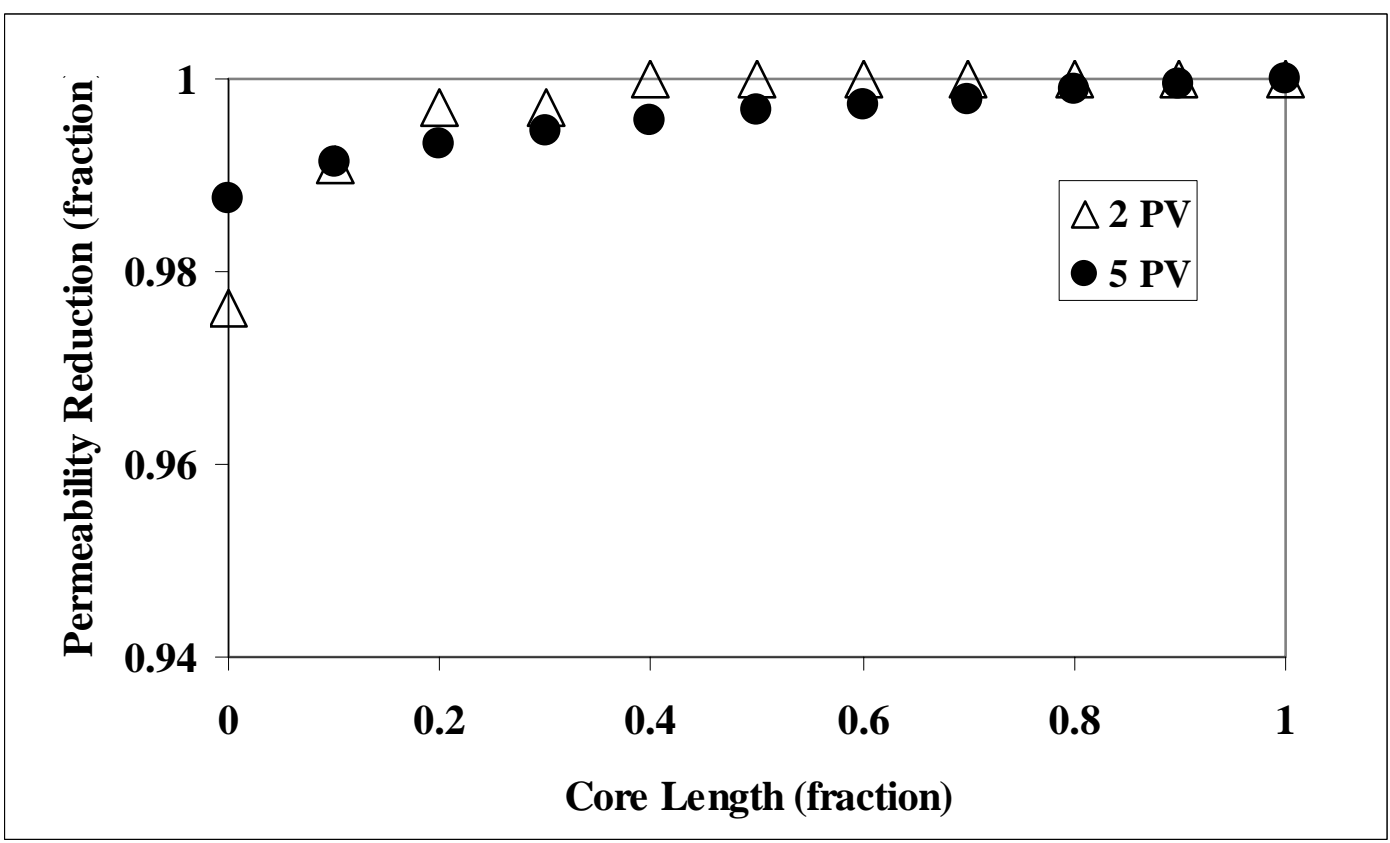

Figure 19. Permeability reduction in Berea cores after 2 and 5 PV injections. 


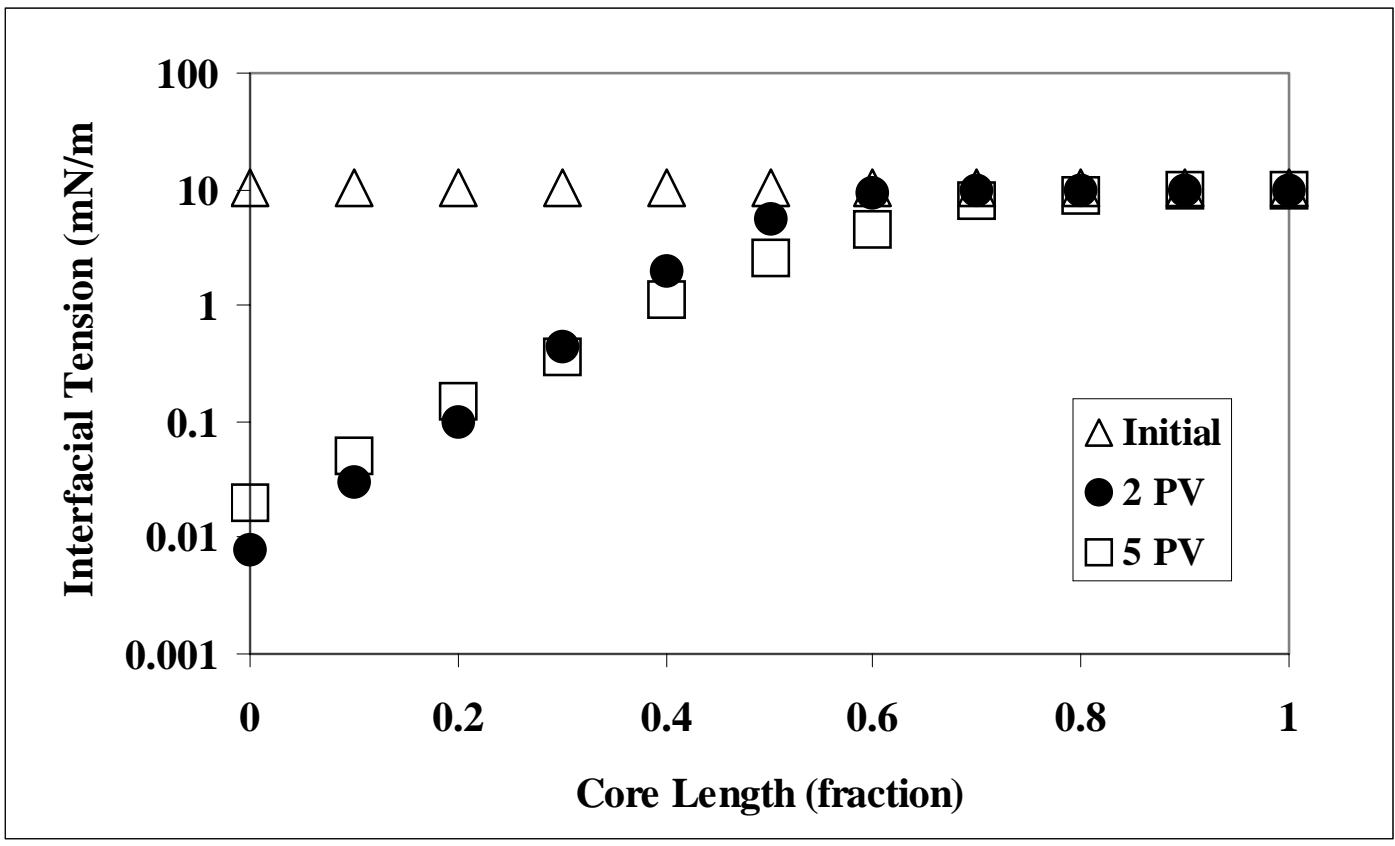

Figure 20. Interfacial tension profiles in Berea cores for different injection volumes.

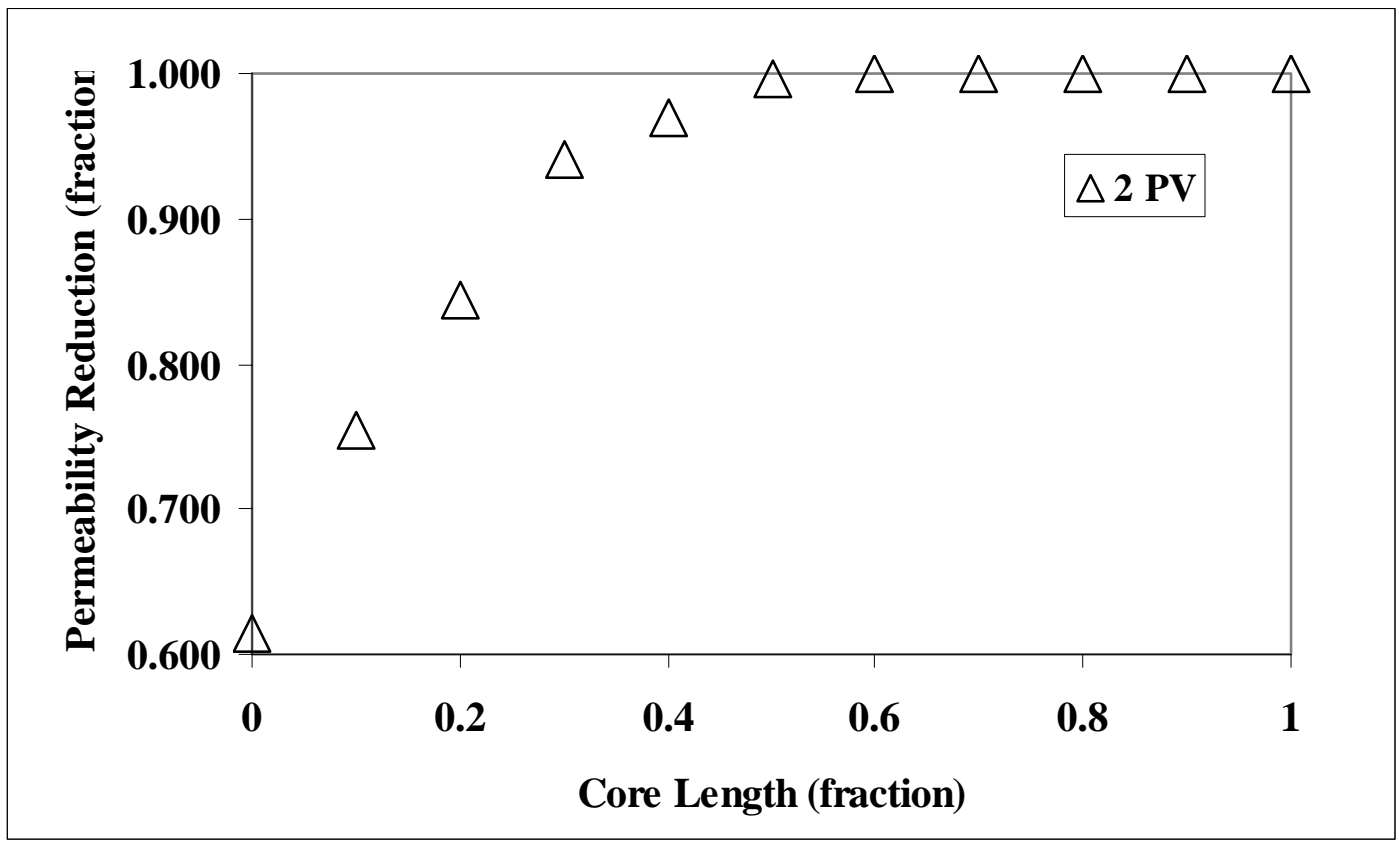

Figure 21. Permeability reduction profile in the Berea core after 2 PV injection for a NIPER 11 culture. 


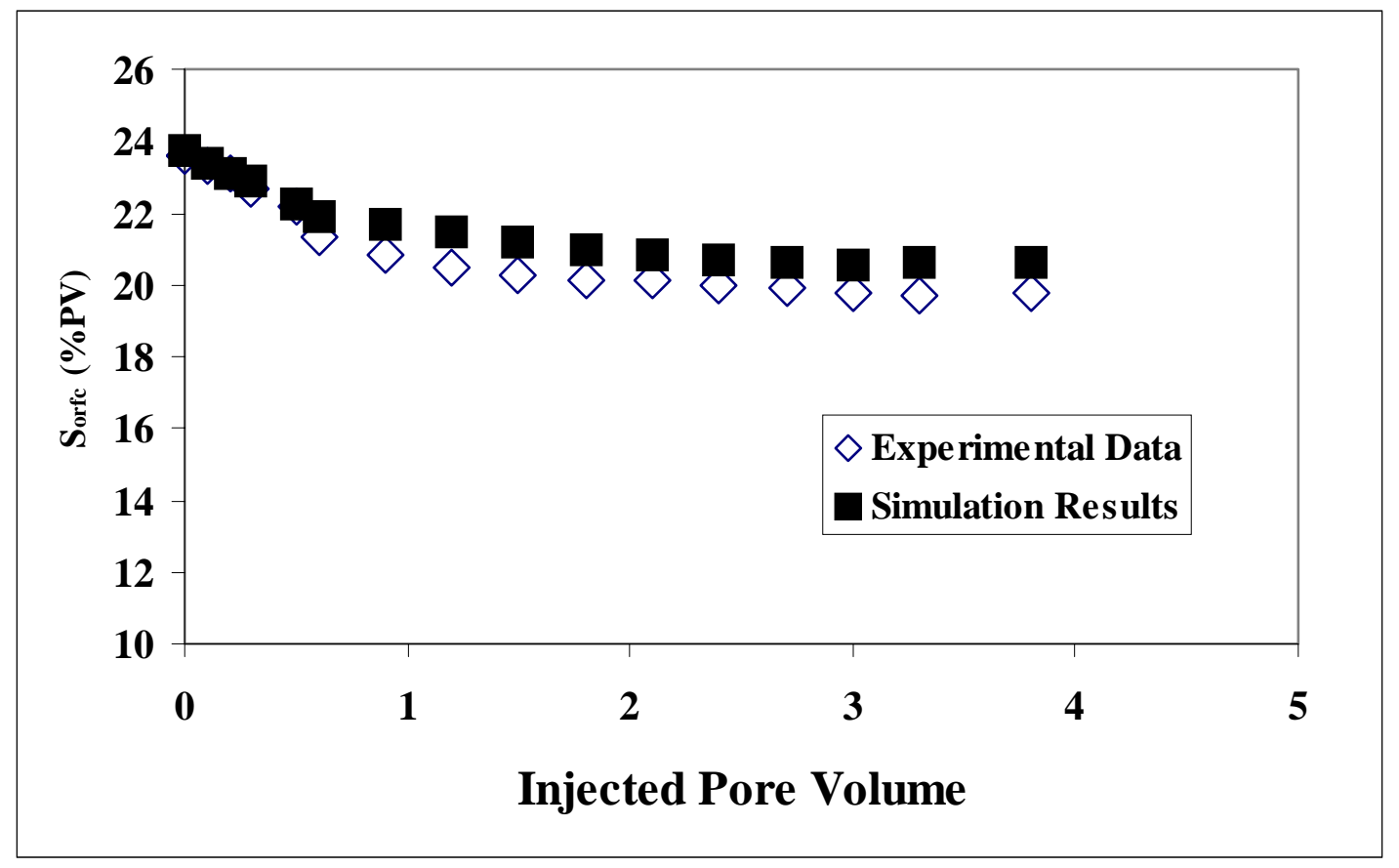

Figure 22. Comparison of experimental and simulation results for oil recovery by NIPER 11.

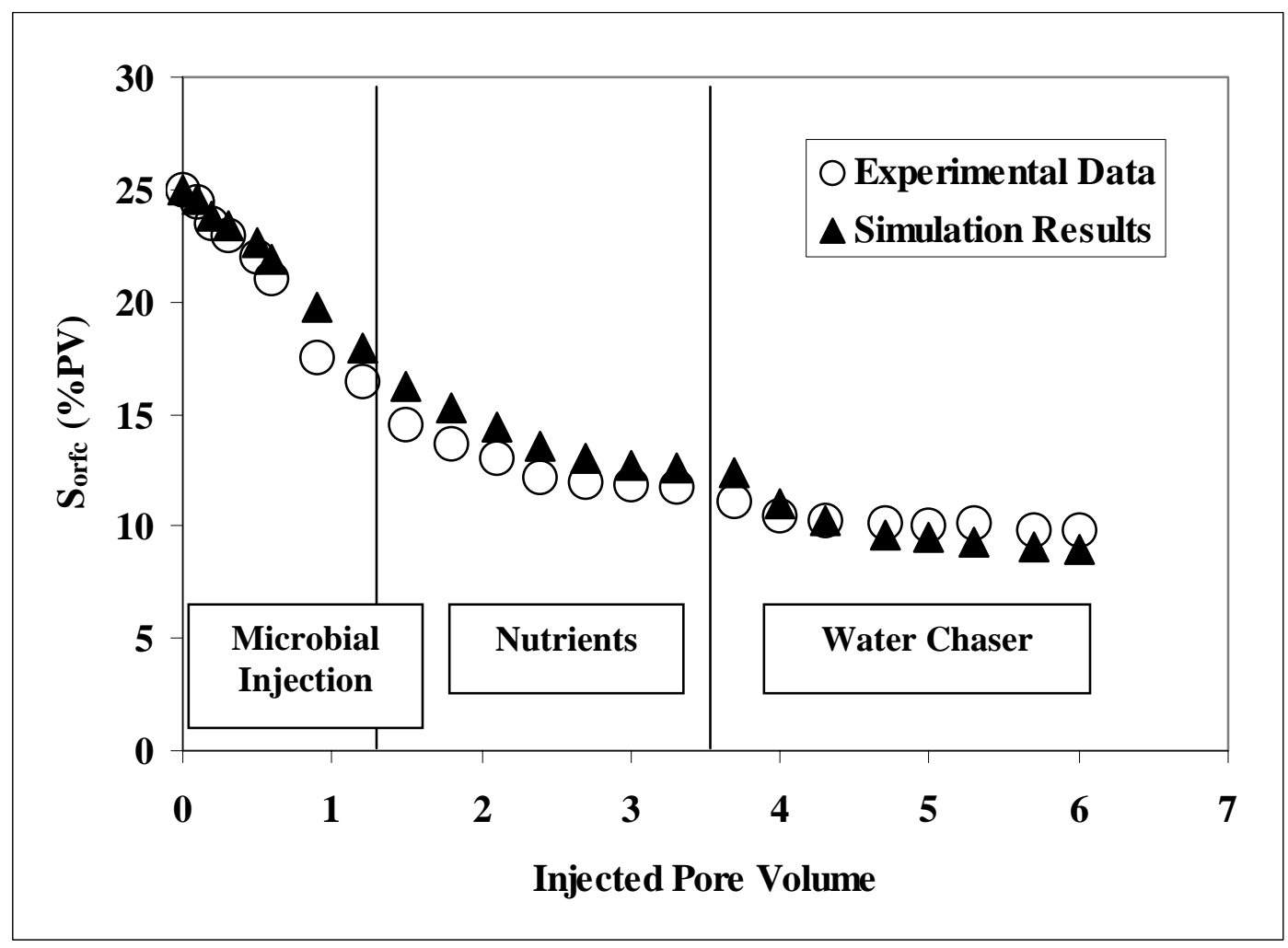

Figure 23. Comparison of experimental and simulation results for oil recovery by NIPER $1 A$. 


\section{COMBINED MiCROBIAL SURFACTANT-POL YMER SYSTEM FOR IMPROVED OIL MOBILITY AND CONFORMANCE CONTROL}

\section{PROJECT INFORMATION}

\section{PUBLICATIONS AND PRESENTATIONS}

Enayet, Sh., and Gabitto, J. F., "Alternative Production of Biosurfactants and Biopolymers by Metabolic Manipulation,” submitted for publication to Canadian Journal of Petroleum Technology, 2005.

Gabitto, J. F., "Combined Microbial Surfactant-Polymer System for Improved Oil Recovery," paper presented at the Annual Meeting of the AIChE , Austin, TX, November 2004.

Eta, O., Rame, M., and Gabitto, J. F., "Combined Microbial Surfactant-Polymer Strategies for Improved Oil Recovery,” paper presented at the Annual Meeting of the AIChE, San Francisco, CA, November 2003.

\section{STUDENTS}

Mr. Oscar Eta participated during 2002 and 2003 in the research work done on this project. Mr. Eta presented a research paper entitled "Combined Microbial Production of Biosurfactants and Biopolymers by Metabolic Manipulation” as part of the requirements to obtain the Master degree on Engineering at Prairie View A\&M University.

Ms. Sharmila Enayet participated during 2004 and 2005 in the experimental research work done on this project. She presented a research paper entitled "Alternative Production of Biosurfactants and Biopolymers by Metabolic Manipulation” as part of the requirements to obtain the Master degree on Engineering at Prairie View A\&M University. 